\title{
ON DESCENDING COHOMOLOGY GEOMETRICALLY
}

\author{
JEFFREY D. ACHTER, SEBASTIAN CASALAINA-MARTIN, AND CHARLES VIAL
}

\begin{abstract}
In this paper, motivated by a problem posed by Barry Mazur, we show that for smooth projective varieties over the rationals, the odd cohomology groups of degree less than or equal to the dimension can be modeled by the cohomology of an abelian variety, provided the geometric coniveau is maximal. This provides an affirmative answer to Mazur's question for all uni-ruled threefolds, for instance. Concerning cohomology in degree three, we show that the image of the Abel-Jacobi map admits a distinguished model over the rationals.
\end{abstract}

\section{INTRODUCTION}

We consider the problem of determining when the cohomology of a smooth projective variety $X$ over a field $K$ can be modeled by an abelian variety. For example, for a prime number $\ell$ different from the characteristic of $K$, it is well known that the first $\ell$-adic cohomology group $H^{1}\left(X_{\bar{K}}, \mathbb{Q}_{\ell}\right)$ can be modeled by the Albanese variety $\operatorname{Alb}(X)$ in the sense that it is isomorphic as a $\operatorname{Gal}(K)$-representation to $H^{1}\left(\operatorname{Alb}(X)_{\bar{K}}, \mathbb{Q}_{\ell}\right)$. Our primary motivation is a special case of a problem posed by Barry Mazur Maz11, Maz14 at the birthday conference for Joe Harris in 2011. To fix ideas, suppose $X$ is a smooth projective variety over a number field $K$. For each prime number $\ell$ and nonnegative integer $n$, the cohomology group $H^{2 n+1}\left(X_{\bar{K}}, \mathbb{Q}_{\ell}\right)$ admits a continuous action by $\operatorname{Gal}(K)$, and $H^{2 n+1}\left(X_{\bar{K}}, \mathbb{Q}_{\ell}(n)\right)$ has weight one. Now further suppose that $h^{p, q}=0$ for $p+q=2 n+1, p, q \neq n$. Thanks to Mazur's Newton over Hodge Theorem [Maz72], the twisted Galois representation $H^{2 n+1}\left(X_{\bar{K}}, \mathbb{Q}_{\ell}(n)\right)$ is still effective; each eigenvalue of Frobenius is actually an algebraic integer. Thus, $H^{2 n+1}\left(X_{\bar{K}}, \mathbb{Q}_{\ell}(n)\right)$ could be the cohomology group of some abelian variety in the sense that there is no obvious obstruction to the existence of an abelian variety $J / K$ such that for all prime numbers $\ell$ there is an isomorphism of $\mathrm{Gal}(K)$-representations

$$
H^{1}\left(J_{\bar{K}}, \mathbb{Q}_{\ell}\right) \cong H^{2 n+1}\left(X_{\bar{K}}, \mathbb{Q}_{\ell}(n)\right) \text {. }
$$

Mazur's question is to determine when such an abelian variety $J / K$ actually exists.

Note that an isomorphism as in (0.1) only characterizes the abelian variety $J / K$ up to isogeny. This ambiguity persists even if one works with $\mathbb{Z}_{\ell}$-cohomology groups. Indeed, there exist elliptic curves $E_{1}$ and $E_{2}$ over a number field such that, for each $\ell$, the Tate modules $T_{\ell} E_{1}$ and $T_{\ell} E_{2}$ are isomorphic as Galois-representations; and yet $E_{1}$ and $E_{2}$ are not isomorphic, even over $\mathbb{C}$ Zar08, Sec. 12]. Following Mazur, the phantom isogeny class for $X$, in degree $n$, is the isogeny class of abelian varieties satisfying (0.1). Any member of this isogeny class will be called a phantom abelian variety for $X$. (We make the analogous definition for varieties over an arbitrary base field.)

This type of question has of course been considered in many contexts, and results in the literature provide a great deal of motivation. To begin, from the arithmetic perspective, the theorems of Tate and Honda [Tat66, Tat71, Hon68] imply (under certain further hypotheses; see \$1.4) that for

Date: October 26, 2016.

The first author was partially supported by grants from the Simons Foundation (204164) and the NSA (H9823014-1-0161, H98230-15-1-0247 and H98230-16-1-0046). The second author was partially supported by NSF grant DMS-1101333 and a Simons Foundation Collaboration Grant for Mathematicians (317572). The third author was supported by the Fund for Mathematics at the Institute for Advanced Study and by EPSRC Early Career Fellowship $\mathrm{EP} / \mathrm{K} 005545 / 1$. 
a prime $\mathfrak{p}$ of good reduction for $X$, there exists an abelian variety $J_{\mathfrak{p}} / \kappa(\mathfrak{p})$ such that, if a phantom abelian variety $J / K$ were to exist, then the reduction of $J$ modulo $\mathfrak{p}$ would be isogenous to $J_{\mathfrak{p}}$. Further motivation comes from complex geometry. Under our hypotheses on the Hodge numbers, the intermediate Jacobian $J^{2 n+1}\left(X_{\mathbb{C}}\right)=H^{n, n+1}\left(X_{\mathbb{C}}\right) / H^{2 n+1}\left(X_{\mathbb{C}}, \mathbb{Z}\right)$ is equal to the algebraic intermediate Jacobian, and is consequently an abelian variety. Since the dual abelian variety $\widehat{J}^{2 n+1}\left(X_{\mathbb{C}}\right)$ satisfies $H^{1}\left(\widehat{J}^{2 n+1}\left(X_{\mathbb{C}}\right), \mathbb{Z}_{\ell}\right)=H^{2 n+1}\left(X_{\mathbb{C}}, \mathbb{Z}_{\ell}(n)\right)$, if the intermediate Jacobian were isogenous to an abelian variety that descended to $K$, this would provide a candidate for a phantom abelian variety. As justification for this approach, recall that a theorem of Deligne Del72] implies that for a complete intersection of odd dimension with Hodge level 1, the intermediate Jacobian descends to $K$ and its dual provides a phantom abelian variety.

In this paper, we establish the existence of phantom abelian varieties for more general smooth projective varieties, but under some further hypotheses on the cohomology. Namely, we make the stronger, yet natural, hypothesis that $H^{2 n+1}\left(X_{\mathbb{C}}, \mathbb{Q}\right)$ has geometric coniveau $n$, meaning that $H^{2 n+1}\left(X_{\mathbb{C}}, \mathbb{Q}\right)$ is supported on a subvariety of $X_{\mathbb{C}}$ of codimension $n$. In fact, assuming the Tate conjecture for $\widehat{J} \times_{K} X$, the geometric coniveau $n$ part is an a priori upper bound on what can be modeled directly with an abelian variety.

Theorem A ((Theorem 2.1)). Suppose $X$ is a smooth projective variety of pure dimension over a field $K \subseteq \mathbb{C}$. If $H^{2 n+1}\left(X_{\mathbb{C}}, \mathbb{Q}\right)$ has geometric coniveau $n$, for some integer $n$ with $2 n+1 \leq \operatorname{dim} X$, then there exist an abelian variety $J$ over $K$, with $J_{\mathbb{C}}$ isogenous to the intermediate Jacobian $J^{2 n+1}\left(X_{\mathbb{C}}\right)$, and a correspondence on $\widehat{J} \times_{K} X$ that induces, for each prime $\ell$, an isomorphism of $\operatorname{Gal}(K)$-representations

$$
H^{1}\left(\widehat{J}_{\bar{K}}, \mathbb{Q}_{\ell}\right) \cong H^{2 n+1}\left(X_{\bar{K}}, \mathbb{Q}_{\ell}(n)\right) .
$$

Moreover, its inverse is induced by a correspondence on $X \times_{K} \widehat{J}$.

We note that the theorem provides an affirmative answer to Mazur's question for all threefolds with $\mathrm{CH}_{0}$ supported on a surface (e.g., for all uni-ruled threefolds ; see Corollary 5.2), and, assuming the generalized Hodge conjecture, for all threefolds with $h^{3,0}=0$. More generally, Theorem 2.1 provides for all $n$ a phantom for the geometric coniveau part $\mathrm{N}^{n} H^{2 n+1}\left(X_{\bar{K}}, \mathbb{Q}_{\ell}(n)\right)$ as soon as $X$ satisfies the Lefschetz standard conjecture.

In Theorem A, operating only under the hypothesis of geometric coniveau $n$, we find a suitable phantom $J / K$ and show that its dual is geometrically isogenous to the intermediate Jacobian $J^{2 n+1}\left(X_{\mathbb{C}}\right)$. This does not imply that $J^{2 n+1}\left(X_{\mathbb{C}}\right)$ itself descends to $K$. In contrast, in the special case $n=1$, we can single out an isomorphism class (in fact, a model over $K$ of the dual of the intermediate Jacobian) within the phantom isogeny class. More generally, without any hypothesis on geometric coniveau, we extend Murre's work Mur85 on algebraic representatives to the setting of non-algebraically closed fields to show (see Corollary 5.2) :

Theorem B. Let $X$ be a smooth projective variety over a field $K \subseteq \mathbb{C}$. The abelian variety $J_{a}^{3}\left(X_{\mathbb{C}}\right)$ that is the image of the Abel-Jacobi map AJ: $A^{2}\left(X_{\mathbb{C}}\right) \rightarrow J^{3}\left(X_{\mathbb{C}}\right)$ has a distinguished model $J$ over $K$ making the Abel-Jacobi map $A J: A^{2}\left(X_{\mathbb{C}}\right) \rightarrow J_{a}^{3}\left(X_{\mathbb{C}}\right)$ Aut $(\mathbb{C} / K)$-equivariant. Moreover, $\hat{J}$ provides a phantom for $\mathrm{N}^{1} H^{3}\left(X_{\bar{K}}, \mathbb{Q}_{\ell}(1)\right)$.

We also establish results regarding specialization of algebraic representatives ( algebraically closed base change of algebraic representatives (Theorem 3.7). As suggested by Lenny Taelman, our results extending Murre's work on algebraic representatives can be interpreted in a functorial framework to provide algebraic representatives over any perfect field. More precisely, let $X$ be a smooth projective variety over a perfect field $K$. Denote by $\mathrm{A}_{\natural}^{2}(X)$ the contravariant functor on the category of smooth integral varieties over $K$ to the category of abelian groups given by families of codimension 2 cycles on $X$ whose geometric fibers are algebraically trivial. Then 
there exist an abelian variety $\mathrm{Ab}^{2}(X)$ over $K$ and a natural transformation of contravariant functors $\mathrm{A}_{\natural}^{2}(X) \rightarrow \mathrm{Ab}^{2}(X)$ that is initial among all natural transformations of contravariant functors $\mathrm{A}_{\natural}^{2}(X) \rightarrow A$ to an abelian variety $A$ over $K$. This approach will appear in ACMVa.

In concomitant work ACMVb, following [Bea77] we provide a geometric construction of the phantom abelian variety when $X$ is an odd-dimensional quadric bundle over a surface over a field of characteristic $\neq 2$. Mazur has also asked for the relationship between phantom abelian varieties and Albaneses of Hilbert and Kontsevich spaces associated to $X$. In future work we will discuss how this question relates to Theorem B.

Outline. In Section 1, we review the notions of geometric, Hodge, and Tate coniveau filtrations and relate the final step of these filtrations on odd-degree cohomology groups with the cohomology of curves, the Abel-Jacobi map, and Mazur's question on phantoms. The case of finite fields is discussed in 1.4. We prove our results on phantom abelian varieties in two parts that complement each other: Theorem A (Theorem 2.1) gives general criteria for the existence of a phantom, and Theorem B (Theorem 5.1 and Corollary 5.2) shows that the image of the second Abel-Jacobi map descends to a field of definition. The proof of Theorem 2.1 relies essentially on polarizations of Hodge structures, carried out in 92 , while the proof of Theorem [5.1] is based on Murre's theory of algebraic representatives [Mur85, and is carried out in 93, 44, In Section 3 we review the results of Murre on algebraic representatives for smooth projective varieties defined over algebraically closed fields and prove in Theorem 3.7 that algebraic representatives, if they exist, behave well under base change along algebraically closed fields. In Section 4 we extend Murre's theory of algebraic representative to the setting of Galois field extensions and prove in Theorem 4.4 that the algebraic representative, if it exists, inherits a Galois descent datum. In Section 5 we prove Theorem 5.1 , providing the connection between algebraic representatives and phantoms. In Section 6 we discuss specialization of algebraic representatives and phantom abelian varieties, as well as some results concerning algebraic representatives for higher codimension cycles.

Acknowledgements. We would like to thank Barry Mazur for his comments and encouragement. We are very grateful to Claire Voisin for explaining the connection between Mazur's question and Murre's work, which directly led to our work on algebraic representatives. We thank the anonymous referees for a number of comments that have strengthened our results, and improved the exposition. In addition, we thank David Grant, Brendan Hassett, Bruno Kahn, Vasudevan Srinivas, Lenny Taelman, Rahbar Virk, Felipe Voloch, and Jonathan Wise for useful conversations. Conventions. A variety over a field is a geometrically reduced separated scheme of finite type over that field. A curve (resp. surface) is a variety of pure dimension 1 (resp. 2). Given a variety $X$ over a field, $\mathrm{CH}^{i}(X)$ denotes the Chow group of codimension $i$ cycles modulo rational equivalence, and $\mathrm{A}^{i}(X) \subseteq \mathrm{CH}^{i}(X)$ denotes the subgroup of cycles algebraically equivalent to 0 . For an abelian variety $A$ over a field $K$, we denote by $\widehat{A}$ the dual abelian variety $\operatorname{Pic}_{A / K}^{0}$.

\section{Phantoms and coniveau filtrations}

The aim of this section is to highlight the relevance of the geometric coniveau filtration in the context of Mazur's question. The main question we will tackle in this work is Question 1.4, where we ask whether the deepest part of the geometric coniveau filtration on odd-degree cohomology groups can be modeled by abelian varieties. To this end we discuss Hodge, Tate, and geometric coniveau filtrations, and their link to the Abel-Jacobi map. One of the key points for later arguments is Proposition 1.3 stating that the deepest part of the geometric coniveau filtration can be realized via the cohomology of a curve.

1.1. Hodge theory and geometric Galois representations. While our motivating problem is posed purely in terms of Galois representations, it has a natural Hodge-theoretic analogue. 
Question 1.1 ([Voi14, Que. 2.43]). Let $X$ be a smooth complex projective variety. Given a weight $i$ Hodge sub-structure $L \subseteq H^{i}(X, \mathbb{Q})$ of Hodge coniveau $r$, so that

$$
L_{\mathbb{C}}=L^{i-r, r} \oplus \cdots \oplus L^{r, i-r},
$$

(and $L(r)$ is an effective weight $i-2 r$ Hodge structure), does there exist a smooth complex projective variety $Y$ admitting a Hodge sub-structure of $L^{\prime} \subseteq H^{i-2 r}(Y, \mathbb{Q})$ with $L^{\prime} \cong L(r)$ ?

In the case that $i=2 n+1$ is odd, and $L$ has Hodge coniveau $n$, the question has an affirmative answer. Indeed, recall that the algebraic intermediate Jacobian, $J_{\text {alg }}^{2 n+1}(X) \subseteq J^{2 n+1}(X)$, is defined to be the largest complex sub-torus with tangent space contained in $H^{n, n+1}(X)$. This is an abelian variety (e.g., VVoi07, p.304]), and clearly $L(n)$ can be obtained as a Hodge sub-structure of $H^{1}\left(\widehat{J}_{\text {alg }}^{2 n+1}, \mathbb{Q}\right)$.

For Mazur's question we are interested in an $\ell$-adic analogue of Question 1.1. Now let $K$ be a field, such as a number field, which is finitely generated over the prime field. A $\operatorname{Gal}(K)$-representation $V_{\ell}$ over $\mathbb{Q}_{\ell}$ has weight $i$ if, for each unramified prime $v$ of $K$ (and each embedding $\overline{\mathbb{Q}}_{\ell} \rightarrow \mathbb{C}$ ), each eigenvalue of Frobenius at $v$ has size $\sqrt{q_{v}^{i}}$, where $q_{v}$ is the cardinality of the residue field at $v$. We say $V_{\ell}$ is entire if all eigenvalues of Frobenius are algebraic integers. We say $V_{\ell}$ is effective of weight $w$ if $V_{\ell}$ and $\operatorname{Hom}\left(V_{\ell}, \mathbb{Q}_{\ell}(-w)\right)$ are entire and of weight $w$. For a smooth projective variety $X$ over $K$, by Poincaré duality and hard Lefschetz, we have $\operatorname{Hom}\left(H^{i}\left(X_{\bar{K}}, \mathbb{Q}_{\ell}\right), \mathbb{Q}_{\ell}(-i)\right) \cong H^{i}\left(X_{\bar{K}}, \mathbb{Q}_{\ell}\right)$, so that a geometric representation $H^{i}\left(X_{\bar{K}}, \mathbb{Q}_{\ell}(\nu)\right.$ ) is effective (of weight $i-2 \nu$ ) if and only if it is entire (e.g., [Jan94, p.269]).

Question 1.2. Let $X$ be a smooth projective variety over a field $K$ that is finitely generated over the prime field. Given a $\mathrm{Gal}(K)$-sub-representation $L_{\ell} \subseteq H^{i}\left(X_{\bar{K}}, \mathbb{Q}_{\ell}\right)$ with the property that $L_{\ell}(r)$ is effective (of weight $i-2 r$ ), does there exist a smooth projective variety $Y$ over $K$ admitting a $\operatorname{Gal}(K)$-sub-representation $L_{\ell}^{\prime} \subseteq H^{i-2 r}\left(Y_{\bar{K}}, \mathbb{Q}_{\ell}\right)$ with $L_{\ell}^{\prime} \cong L_{\ell}(r)$ ?

Unlike the case of Hodge structures, even when $i=2 n+1$ is odd, and $L_{\ell}(n)$ is effective of weight 1 , it is not known exactly when Question 1.2 will have an affirmative answer. Theorem $\mathrm{A}$ provides an affirmative answer under further hypotheses on the geometric coniveau; we review coniveau in the sections below. (See 1.4 for a discussion of the connection between Question 1.2 over finite fields and the theory of Honda-Tate; see ACMVc for a discussion of the extent to which $Y$ may be taken to be geometrically integral.)

1.2. Coniveau filtrations and the generalized Hodge and Tate conjectures. Questions 1.1 and 1.2 are related to the generalized Hodge and Tate conjectures (Gro68]). Our treatment here follows [Jan94, §3]. Let $X$ be a smooth projective variety over a field $K$. The geometric coniveau filtration $\mathrm{N}^{\nu} H^{i}\left(X_{\bar{K}}, \mathbb{Q}_{\ell}\right)$ is defined by:

$$
\mathrm{N}^{\nu} H^{i}\left(X_{\bar{K}}, \mathbb{Q}_{\ell}\right)=\sum_{\substack{Z \subseteq X \\ \text { closed, codim } \geq \nu}} \operatorname{Ker}\left(H^{i}\left(X_{\bar{K}}, \mathbb{Q}_{\ell}\right) \rightarrow H^{i}\left(X_{\bar{K}} \backslash Z_{\bar{K}}, \mathbb{Q}_{\ell}\right)\right) .
$$

By resolution of singularities and by Deligne's theory of mixed Hodge structures when char $K=0$, or by de Jong's theory of alterations and by the Weil conjectures when $K$ is a finite field, the geometric coniveau filtration can also be characterized by the following formula (see [1106, Sec. $4.4(\mathrm{~d})])$ :

$$
\mathrm{N}^{\nu} H^{i}\left(X_{\bar{K}}, \mathbb{Q}_{\ell}\right)=\sum_{\substack{f: Y \rightarrow X \\ \operatorname{dim} Y \text { dim } X-\nu \\ Y \text { smooth proj }}} \operatorname{Im}\left(f_{*}: H^{i-2 \nu}\left(Y_{\bar{K}}, \mathbb{Q}_{\ell}(-\nu)\right) \rightarrow H^{i}\left(X_{\bar{K}}, \mathbb{Q}_{\ell}\right)\right) .
$$

Now suppose that $K$ is a sub-field of $\mathbb{C}$. The geometric coniveau filtration on Betti cohomology $\mathrm{N}^{\nu} H^{i}\left(X_{\mathbb{C}}, \mathbb{Q}\right)$ is defined in the same way. Note that usually a class $\alpha \in H^{i}\left(X_{\mathbb{C}}, \mathbb{Q}\right)$ is said to 
have geometric coniveau $\nu$ if it vanishes when restricted to the complement of a closed complex subvariety of $X_{\mathbb{C}}$ of pure codimension $\nu$. However, using a standard argument involving spreading out, specialization, and then Galois averaging, it suffices as indicated above to consider projective varieties $Z$, and smooth projective varieties $Y$, of pure dimension $\operatorname{dim} X-\nu$ defined over $K$.

By virtue of the fact that the canonical comparison isomorphisms between Betti, de Rham and étale cohomologies are compatible with Gysin maps, and even cycle maps [Del82, §1], there are canonical isomorphisms

$$
\left(\mathrm{N}^{\nu}\left(H^{i}\left(X_{\mathbb{C}}, \mathbb{Q}\right)\right)\right) \otimes_{\mathbb{Q}} \mathbb{Q}_{\ell} \cong \mathrm{N}^{\nu} H^{i}\left(X_{\bar{K}}, \mathbb{Q}_{\ell}\right),
$$

which justify our apparent abuse of notation. The generalized Hodge and Tate conjectures relate these geometric coniveau filtrations to two other filtrations.

Call a $\mathbb{Q}$-Hodge structure $V$ effective if $V^{p, q}=0$ unless $p, q \geq 0$. The Hodge coniveau filtration $\mathrm{N}_{H}^{\nu}$ of $H^{i}\left(X_{\mathbb{C}}, \mathbb{Q}\right)$ has term $\mathrm{N}_{H}^{\nu} H^{i}\left(X_{\mathbb{C}}, \mathbb{Q}\right)$ defined to be the span of all Hodge sub-structures $V \subseteq H^{i}\left(X_{\mathbb{C}}, \mathbb{Q}\right)$ such that $V(\nu)$ is effective, i.e., such that $V^{p, q}=0$ unless $p, q \geq \nu$. The Gysin map provides a natural inclusion

$$
\mathrm{N}^{\nu} H^{i}\left(X_{\mathbb{C}}, \mathbb{Q}\right) \subseteq \mathrm{N}_{H}^{\nu} H^{i}\left(X_{\mathbb{C}}, \mathbb{Q}\right) ;
$$

the generalized Hodge conjecture (for $X$, in degree $i$ ) asserts equality in (1.4).

Similarly, if $K$ is finitely generated over the prime field, define a filtration $\mathrm{N}_{\ell}^{\nu} H^{i}\left(X_{\bar{K}}, \mathbb{Q}_{\ell}\right)$ as the span of all sub-Gal $(K)$-representations $V_{\ell} \subset H^{i}\left(X_{\bar{K}}, \mathbb{Q}_{\ell}\right)$ such that $V_{\ell}(\nu)$ is effective (of weight $i-2 \nu$ ). Again, the Gysin map (this time, for étale cohomology) provides a natural inclusion

$$
\mathrm{N}^{\nu} H^{i}\left(X_{\bar{K}}, \mathbb{Q}_{\ell}\right) \subseteq \mathrm{N}_{\ell}^{\nu} H^{i}\left(X_{\bar{K}}, \mathbb{Q}_{\ell}\right),
$$

and the generalized Tate conjecture (for $X$, in degree $i$ ) asserts equality in (1.5). Sometimes, we will abuse notation slightly and denote the $r$-th twist of step $\nu$ in the geometric coniveau filtration by

$$
\mathrm{N}^{\nu} H^{i}\left(X_{\bar{K}}, \mathbb{Q}_{\ell}(r)\right):=\left(\mathrm{N}^{\nu} H^{i}\left(X_{\bar{K}}, \mathbb{Q}_{\ell}\right)\right) \otimes \mathbb{Q}_{\ell}(r) ;
$$

we employ similar notation for the other filtrations (also on Betti cohomology).

Important to us will be the following characterization of the final step of the geometric coniveau filtration for odd-degree cohomology groups :

Proposition 1.3 ([Via13, §1.2.3]). Suppose $X$ is a smooth projective variety over a field $K$, which is either of characteristic zero or finite, and let $\ell \neq \operatorname{char}(K)$ be a prime. Let $n$ be $a$ non-negative integer. Then there exist a smooth projective curve $C$ over $K$ and a correspondence $\gamma \in \mathrm{CH}^{n+1}\left(C \times_{K} X\right) \otimes_{\mathbb{Z}} \mathbb{Q}$ such that the induced morphism of $\operatorname{Gal}(K)$-representations

$$
\gamma_{*}: H^{1}\left(C_{\bar{K}}, \mathbb{Q}_{\ell}\right) \rightarrow H^{2 n+1}\left(X_{\bar{K}}, \mathbb{Q}_{\ell}(n)\right)
$$

has image $\mathrm{N}^{n} H^{2 n+1}\left(X_{\bar{K}}, \mathbb{Q}_{\ell}(n)\right)$. If $K \subseteq \mathbb{C}$, then the morphism of Hodge structures

$$
\gamma_{*}: H^{1}\left(C_{\mathbb{C}}, \mathbb{Q}\right) \rightarrow H^{2 n+1}\left(X_{\mathbb{C}}, \mathbb{Q}(n)\right)
$$

has image $\mathrm{N}^{n} H^{2 n+1}\left(X_{\mathbb{C}}, \mathbb{Q}(n)\right)$. Moreover, $\mathrm{N}^{n} H^{2 n+1}\left(X_{\bar{K}}, \mathbb{Q}_{\ell}(n)\right)$ is a semisimple representation of $\operatorname{Gal}(K)$.

Proof. This is proved in Via13] in the case of Betti cohomology (see the formula $\widetilde{\mathrm{N}}^{\lfloor i / 2\rfloor} H_{i}(X)=$ $\mathrm{N}^{\lfloor i / 2\rfloor} H_{i}(X)$ in $\$ 1.2 .3$ therein). The proof adapts to our more general setting: up to working component-wise, we may and do assume that $X$ is connected, say of dimension $d$. Since $H^{2 n+1}\left(X_{\bar{K}}, \mathbb{Q}_{\ell}(n)\right)$ is a finite-dimensional $\mathbb{Q}_{\ell}$-vector space, from the characterization of coniveau given in (1.2) there exist a smooth projective variety $Y$ of dimension $d_{Y}=d-n$ over $K$ and a $K$-morphism $f: Y \rightarrow X$ such that

$$
\mathrm{N}^{n} H^{2 n+1}\left(X_{\bar{K}}, \mathbb{Q}_{\ell}(n)\right)=\operatorname{Im}\left(f_{*}: H_{5}^{1}\left(Y_{\bar{K}}, \mathbb{Q}_{\ell}\right) \rightarrow H^{2 n+1}\left(X_{\bar{K}}, \mathbb{Q}_{\ell}(n)\right)\right) .
$$


By Bertini Poo04, let $\iota: C \hookrightarrow Y$ be a one-dimensional smooth linear section of $Y$. The hard Lefschetz theorem [Del80, Thm. 4.1.1] states that intersecting with $C$ yields an isomorphism

$$
\iota_{*} \iota^{*}: H^{1}\left(Y_{\bar{K}}, \mathbb{Q}_{\ell}\right) \hookrightarrow H^{1}\left(C_{\bar{K}}, \mathbb{Q}_{\ell}\right) \rightarrow H^{2 d_{Y}-1}\left(Y_{\bar{K}}, \mathbb{Q}_{\ell}\left(d_{Y}-1\right)\right)
$$

The Lefschetz Standard Conjecture is known for $\ell$-adic cohomology and for Betti cohomology in degree $\leq 1$ (see [Kle68, Thm. 2A9(5)]), meaning in our case that the map $\left(\iota_{*} \iota^{*}\right)^{-1}$ is induced by a correspondence $\Lambda \in \mathrm{CH}^{1}\left(Y \times_{K} Y\right)_{\mathbb{Q}}$. Therefore, the composition

$$
H^{1}\left(C_{\bar{K}}, \mathbb{Q}_{\ell}\right) \stackrel{\iota_{*}}{\longrightarrow} H^{2 d_{Y}-1}\left(Y_{\bar{K}}, \mathbb{Q}_{\ell}\left(d_{Y}-1\right)\right) \stackrel{\Lambda_{*}}{\cong} H^{1}\left(Y_{\bar{K}}, \mathbb{Q}_{\ell}\right) \stackrel{f_{*}}{\longrightarrow} H^{2 n+1}\left(X_{\bar{K}}, \mathbb{Q}_{\ell}(n)\right),
$$

which has image $\mathrm{N}^{n} H^{2 n+1}\left(X_{\bar{K}}, \mathbb{Q}_{\ell}(n)\right)$, is induced by the required correspondence.

That $\mathrm{N}^{n} H^{2 n+1}\left(X_{\bar{K}}, \mathbb{Q}_{\ell}(n)\right)$ is a semisimple representation of $\mathrm{Gal}(K)$ follows immediately from the fact that $H^{1}\left(C_{\bar{K}}, \mathbb{Q}_{\ell}\right)$ is semisimple if $K$ is a field which is finitely generated (over $\mathbb{Q}$ [Fal83, $\mathrm{FWG}^{+} 92$, or a finite field [Tat66, Zar75]).

When $K \subseteq \mathbb{C}$, the comparison isomorphisms (1.3) establish that the image of the induced morphism of Hodge structures $\gamma_{*}: H^{1}\left(C_{\mathbb{C}}, \mathbb{Q}\right) \rightarrow H^{2 n+1}\left(X_{\mathbb{C}}, \mathbb{Q}(n)\right)$ is $\mathrm{N}^{n} H^{2 n+1}\left(X_{\mathbb{C}}, \mathbb{Q}(n)\right)$.

1.3. Coniveau filtrations and the Abel-Jacobi map. In this section we review the connection between the coniveau filtrations and the Abel-Jacobi map. Recall that, for a smooth complex projective variety $X$, the intermediate Jacobian is a complex torus defined by

$$
J^{2 n+1}(X):=F^{n+1} H^{2 n+1}(X, \mathbb{C}) \backslash H^{2 n+1}(X, \mathbb{C}) / H^{2 n+1}(X, \mathbb{Z}),
$$

and that there is an Abel-Jacobi map

$$
\operatorname{Ker}\left(\mathrm{CH}^{n}(X) \rightarrow H^{2 n}(X, \mathbb{Z})\right) \rightarrow J^{2 n+1}\left(X_{\mathbb{C}}\right) .
$$

By definition the algebraic intermediate Jacobian $J_{\text {alg }}^{2 n+1}(X)$ is the largest sub-torus of $J^{2 n+1}(X)$ that is projective; it determines the $n$-th piece of the Hodge coniveau filtration:

$$
\begin{aligned}
\mathrm{N}_{H}^{n} H^{2 n+1}(X, \mathbb{Q}) \otimes_{\mathbb{Q}} \mathbb{C} & =T_{0} J_{\text {alg }}^{2 n+1}(X) \oplus \overline{T_{0} J_{\text {alg }}^{2 n+1}(X)} \\
\mathrm{N}_{H}^{n} H^{2 n+1}(X, \mathbb{Q}(n)) & =H^{1}\left(\widehat{J}_{\text {alg }}^{2 n+1}(X), \mathbb{Q}\right) .
\end{aligned}
$$

On the other hand, it is standard [Voi07, Th. 12.17] that the $n$-th piece of the geometric coniveau filtration $\mathrm{N}^{n} H^{2 n+1}(X, \mathbb{Q})$ is determined by the image of the Abel-Jacobi map. More precisely, the image $J_{a}^{2 n+1}(X)$ of the Abel-Jacobi map $A J: \mathrm{A}^{n+1}(X) \rightarrow J^{2 n+1}(X)$ has tangent space $T_{0} J_{a}^{2 n+1}(X)=H^{n, n+1}(X) \cap \mathrm{N}^{n} H^{2 n+1}(X, \mathbb{C})$, and

$$
\begin{aligned}
\mathrm{N}^{n} H^{2 n+1}(X, \mathbb{Q}) \otimes_{\mathbb{Q}} \mathbb{C} & =T_{0} J_{a}^{2 n+1}(X) \oplus \overline{T_{0} J_{a}^{2 n+1}(X)} \\
\mathrm{N}^{n} H^{2 n+1}(X, \mathbb{Q}(n)) & =H^{1}\left(\widehat{J}_{a}^{2 n+1}(X), \mathbb{Q}\right) .
\end{aligned}
$$

Thus the Abel-Jacobi map restricted to algebraically trivial cycles

$$
A J: \mathrm{A}^{n+1}(X) \rightarrow J^{2 n+1}(X)
$$

is surjective if and only if $H^{2 n+1}(X, \mathbb{Q})$ has geometric coniveau $n$.

In light of this discussion, the first goal of this paper is to consider analogous questions for geometric Galois representations :

Question 1.4. Given a smooth projective variety $X$ over a field $K$ finitely generated over the prime field, do there exist abelian varieties $\tilde{J}$ and $J$ defined over $K$ such that for each prime $\ell$,

$$
\begin{aligned}
& \mathrm{N}_{\ell}^{n} H^{2 n+1}\left(X_{\bar{K}}, \mathbb{Q}_{\ell}(n)\right) \cong H^{1}\left(\tilde{J}_{\bar{K}}, \mathbb{Q}_{\ell}\right) \\
& \mathrm{N}^{n} H^{2 n+1}\left(X_{\bar{K}}, \mathbb{Q}_{\ell}(n)\right) \cong H^{1}\left(J_{\bar{K}}, \mathbb{Q}_{\ell}\right)
\end{aligned}
$$

as $\operatorname{Gal}(K)$-modules? 
The abelian variety $\tilde{J} / K$ would provide an answer to the special case of Question 1.2 that we are considering in this paper, and consequently to Mazur's question (i.e., the case where $\left.\mathrm{N}_{\ell}^{n} H^{2 n+1}\left(X_{\bar{K}}, \mathbb{Q}_{\ell}(n)\right)=H^{2 n+1}\left(X_{\bar{K}}, \mathbb{Q}_{\ell}(n)\right)\right)$. Note that if $\tilde{J}$ exists, then a direct consequence of the Tate conjecture for $X \times_{K} \tilde{J}$ would be that $\mathrm{N}_{\ell}^{n}$ and $\mathrm{N}^{n}$ agree on $H^{2 n+1}\left(X_{\bar{K}}, \mathbb{Q}_{\ell}(n)\right.$ ) (see also [Voi14, p.34] for the corresponding Hodge-theoretic statement). Our aim in this paper is to construct the abelian variety $J / K$, under some further hypotheses.

1.4. Mazur's question over finite fields. Honda-Tate theory suggests an approach to Mazur's question over finite fields. Consider a smooth projective variety $X / \mathbb{F}_{q}$ such that $h^{2 n+1-j, j}(X)=0$ unless $j \in\{n, n+1\}$. By Mazur's "Newton over Hodge" theorem [Maz72], each eigenvalue of Frobenius, acting on $H^{2 n+1}\left(X_{\overline{\mathbb{F}}_{q}}, \mathbb{Q}_{\ell}\right)$, is divisible by $q^{n}$, and thus $H^{2 n+1}\left(X_{\overline{\mathbb{F}}_{q}}, \mathbb{Q}_{\ell}(n)\right)$ is an effective $\operatorname{Gal}\left(\mathbb{F}_{q}\right)$-representation of weight one. It is then natural to ask if there is an abelian variety $J / \mathbb{F}_{q}$ such that $H^{1}\left(J_{\bar{F}_{q}}, \mathbb{Q}_{\ell}\right) \cong H^{2 n+1}\left(X_{\overline{\mathbb{F}}_{q}}, \mathbb{Q}_{\ell}(n)\right)$ as Galois representations. (More generally, of course, one has Question [1.2) Honda-Tate theory asserts a bijection

$\{$ conjugacy classes of $q$-Weil numbers $\} \stackrel{\sim}{\longrightarrow}$ isogeny classes of simple abelian varieties $\left./ \mathbb{F}_{q}\right\}$

which we crudely denote $\varpi \mapsto A_{\varpi}$. The Galois representation attached to an abelian variety over a finite field is semisimple [Tat66], and one immediately deduces from the above bijection that if $V_{\ell}$ is a semisimple effective weight one $\mathbb{Q}_{\ell}$-representation of $\operatorname{Gal}\left(\mathbb{F}_{q}\right)$, then there exist an abelian variety $A / \mathbb{F}_{q}$ and an inclusion of $\operatorname{Gal}\left(\mathbb{F}_{q}\right)$-representations

$$
V_{\ell} \longleftrightarrow H^{1}\left(A_{\bar{F}_{q}}, \mathbb{Q}_{\ell}\right) \text {. }
$$

By starting with a Weil number $\varpi$ for which $[\mathbb{Q}(\varpi): \mathbb{Q}]<2 \operatorname{dim} A_{\varpi}$ (see, e.g., [Tat71, p. 98] for examples), it is easy to construct semisimple representations $V_{\ell}$ for which (1.7) is never an isomorphism. Nonetheless, we have :

Lemma 1.5. Let $X / \mathbb{F}_{q}$ be a smooth projective variety. Suppose that $X$ is of geometric coniveau $n$ in degree $2 n+1$.

(a) There is an abelian variety $A / \mathbb{F}_{q}$ such that, for all $\ell \neq \operatorname{char}\left(\mathbb{F}_{q}\right)$, there is an inclusion of Galois representations

$$
H^{2 n+1}\left(X_{\overline{\mathbb{F}}_{q}}, \mathbb{Q}_{\ell}(n)\right) \longleftrightarrow H^{1}\left(A_{\overline{\mathbb{F}}_{q}}, \mathbb{Q}_{\ell}\right) .
$$

(b) If $X$ is ordinary (the Newton and Hodge polygons in degree $2 n+1$ coincide), or if the Tate conjecture holds for $X \times_{\mathbb{F}_{q}} X$, then one may choose $A$ so that (1.8) is an isomorphism.

Proof. Part (a) follows immediately from Honda-Tate theory and from the semisimplicity of the Galois representation $H^{2 n+1}\left(X_{\bar{F}_{q}}, \mathbb{Q}_{\ell}(n)\right)$ (see Proposition 1.3$)$.

For part (b), first suppose that $X$ is ordinary in degree $2 n+1$. It suffices to verify that for each Weil number $\varpi$ occurring as an eigenvalue of Frobenius in $H^{2 n+1}\left(X_{\overline{\mathbb{F}}_{q}}, \mathbb{Q}_{\ell}(n)\right)$, one has $[\mathbb{Q}(\varpi)$ : $\mathbb{Q}]=2 \operatorname{dim} A_{\varpi}$. By ordinarity, all slopes of the Newton polygon of $H^{2 n+1}\left(X_{\bar{F}_{q}}, \mathbb{Q}_{\ell}(n)\right)$ are 0 and 1. For each $\varpi$, the calculation of $\operatorname{End}\left(A_{\varpi}\right)$ in $\left[\right.$ Tat71, Thm. 1] implies that $[\mathbb{Q}(\varpi): \mathbb{Q}]=2 \operatorname{dim} A_{\varpi}$.

Second, suppose instead that the Tate conjecture holds for $X \times_{\mathbb{F}_{q}} X$. Then one can calculate [Mil94, Prop. 2.4 and 2.8] the dimension of (the motive corresponding to) $H^{2 n+1}\left(X_{\bar{F}_{q}}, \mathbb{Q}_{\ell}(n)\right)$. The calculation of this dimension, which relies only on the Weil numbers, is the same expression used to calculate the dimension of an abelian variety with these Weil numbers. In particular, (1.8) is an isomorphism. 
Remark 1.6. In a similar spirit (but necessarily more involved fashion), Volkov Vol05] has characterized the potentially crystalline $p$-adic representations of $\operatorname{Gal}\left(\mathbb{Q}_{p}\right)$ which arise as summands of the $p$-adic Tate module of an abelian variety over $\mathbb{Q}_{p}$. As with finite fields, this lets one conclude that if $X / \mathbb{Q}_{p}$ is a smooth projective threefold with $h^{3,0}(X)=0$, and if $X$ acquires good reduction over a tamely ramified extension of $\mathbb{Q}_{p}$, then $H^{3}\left(X_{\overline{\mathbb{Q}}_{p}}, \mathbb{Q}_{p}(1)\right)$ is isomorphic to a sub-representation of $H^{1}\left(A_{\overline{\mathbb{Q}}_{p}}, \mathbb{Q}_{p}\right)$ for some abelian variety $A / \mathbb{Q}_{p}$.

\section{Phantoms Via CORRESPOndences And polarizations}

In this section we prove the following theorem, which implies both the first part of Theorem $\mathrm{A}$. and a partial, affirmative answer to Question 1.4. We will write $\mathrm{CH}^{*}(-)_{\mathbb{Q}}$ for $\mathrm{CH}^{*}(-) \otimes_{\mathbb{Z}} \mathbb{Q}$.

Theorem 2.1. Suppose $X$ is a smooth projective variety of pure dimension d over a field $K \subseteq \mathbb{C}$. Let $n$ be a non-negative integer. Then there exist a smooth (possibly reducible) projective curve $C$ over $K$, and a correspondence $\gamma \in \mathrm{CH}^{n+1}\left(C \times_{K} X\right)_{\mathbb{Q}}$, such that for all prime numbers $\ell$ the induced map

$$
\gamma_{*}: H^{1}\left(\left(J_{C / K}\right)_{\bar{K}}, \mathbb{Q}_{\ell}\right) \rightarrow H^{2 n+1}\left(X_{\bar{K}}, \mathbb{Q}_{\ell}(n)\right)
$$

has image $\mathrm{N}^{n} H^{2 n+1}\left(X_{\bar{K}}, \mathbb{Q}_{\ell}(n)\right)$. Assume further any of the following:

(a) $2 n+1 \leq 3$;

(b) $X$ satisfies the Lefschetz standard conjecture;

(c) $2 n+1 \leq d$ and $H^{2 n+1}\left(X_{\mathbb{C}}, \mathbb{Q}(n)\right)$ is of geometric niveau $n-1$, in the sense of Via13, i.e., there exists a (possibly reducible) smooth projective 3 -fold $Y$ over $K$ and a correspondence $\Gamma \in \mathrm{CH}^{n+2}\left(Y \times_{K} X\right)_{\mathbb{Q}}$ such that $\Gamma_{*}: H^{3}\left(Y_{\mathbb{C}}, \mathbb{Q}(1)\right) \rightarrow H^{2 n+1}\left(X_{\mathbb{C}}, \mathbb{Q}(n)\right)$ is surjective;

(d) $2 n+1 \leq d$ and $H^{2 n+1}\left(X_{\mathbb{C}}, \mathbb{Q}(n)\right)$ has geometric coniveau $n$.

Then there exists a sub-abelian variety $J \stackrel{\iota}{\hookrightarrow} J_{C / K}$ defined over $K$ such that for all prime numbers $\ell$, the correspondence $\gamma$ composed with the graph of the inclusion $\Gamma_{\iota}$ induces a split inclusion of $\mathrm{Gal}(K)$-representations

$$
H^{1}\left(J_{\bar{K}}, \mathbb{Q}_{\ell}\right) \stackrel{\gamma_{*} \circ \Gamma_{\iota *}}{\longrightarrow} H^{2 n+1}\left(X_{\bar{K}}, \mathbb{Q}_{\ell}(n)\right),
$$

with the splitting induced by a correspondence over $K$ and with image $\mathrm{N}^{n} H^{2 n+1}\left(X_{\bar{K}}\right.$, $\left.\mathbb{Q}_{\ell}(n)\right)$. In particular, if $2 n+1 \leq d$ and if $H^{2 n+1}\left(X_{\mathbb{C}}, \mathbb{Q}(n)\right)$ is of geometric coniveau $n$, then (2.1) is an isomorphism.

Remark 2.2. Let $X / K$ be a smooth integral projective variety. Let $L / K$ be a finite extension such that $X_{L}$ is a disjoint union of geometrically irreducible components, and $\operatorname{Gal}(L / K)$ acts transitively on these components. If $\mathrm{N}^{n} H^{2 n+1}\left(-, \mathbb{Q}_{\ell}(n)\right)$ of some component of $X_{L}$ admits a phantom abelian variety $A$ over $L$, then $\mathrm{N}^{n} H^{2 n+1}\left(X_{\bar{K}}, \mathbb{Q}_{\ell}(n)\right)$ admits the Weil restriction $\mathbf{R}_{L / K}(A)$ of $A$ over $K$ as a phantom, since $H^{1}\left(\mathbf{R}_{L / K}(A)_{\bar{K}}, \mathbb{Q}_{\ell}\right) \cong \operatorname{Ind}_{\operatorname{Gal}(L)}^{\operatorname{Gal}(K)} H^{1}\left(A_{\bar{K}}, \mathbb{Q}_{\ell}\right)$.

Proof of Theorem [2.1. Proposition 1.3 provides a smooth projective curve $C / K$ and a correspondence $\gamma \in \mathrm{CH}^{n+1}\left(C \times_{K} X\right)_{\mathbb{Q}}$ such that the induced morphism of Hodge structures

$$
\gamma_{*}: H^{1}\left(C_{\mathbb{C}}, \mathbb{Q}\right) \rightarrow H^{2 n+1}\left(X_{\mathbb{C}}, \mathbb{Q}(n)\right)
$$

has image $\mathrm{N}^{n} H^{2 n+1}\left(X_{\mathbb{C}}, \mathbb{Q}(n)\right)$. This establishes the first claim of Theorem 2.1.

In order to prove the remaining claims of Theorem 2.1, we would like to find an abelian subvariety $J / K \hookrightarrow J_{C / K}$ so that the composition

$$
H^{1}\left(J_{\bar{K}}, \mathbb{Q}_{\ell}\right) \longleftrightarrow H^{1}\left(\left(J_{C / K}\right)_{\bar{K}}, \mathbb{Q}_{\ell}\right) \stackrel{\gamma_{*}}{\longrightarrow} \mathrm{N}^{n} H^{2 n+1}\left(X_{\bar{K}}, \mathbb{Q}_{\ell}(n)\right)
$$


is an isomorphism. At the level of rational Hodge structures and complex abelian varieties, this is elementary; the issue is to find a suitable abelian variety defined over $K$. Rather than trying to directly descend an abelian variety from $\mathbb{C}$ to $K$, our strategy for obtaining $J / K$ is motivic in nature and consists in finding a correspondence $\pi \in \mathrm{CH}^{1}\left(C \times_{K} C\right)_{\mathbb{Q}}$ which acts idempotently on $H^{1}\left(C_{\mathbb{C}}, \mathbb{Q}\right)$ such that $\pi_{*} H^{1}\left(C_{\mathbb{C}}, \mathbb{Q}\right)$ is mapped via $\gamma_{*}$ isomorphically onto $\mathrm{N}^{n} H^{2 n+1}\left(X_{\mathbb{C}}, \mathbb{Q}(n)\right)$. Such a correspondence $\pi$ defines a motive $(C, \pi)$, which determines an abelian variety $J / K$; more concretely, the correspondence $\pi$ induces a $K$-morphism $\Pi: J_{C / K} \rightarrow J_{C / K}$, and the image is the desired abelian variety $J / K$ with $H^{1}\left(J_{\bar{K}}, \mathbb{Q}_{\ell}\right)$ identified with $\pi_{*} H^{1}\left(C_{\bar{K}}, \mathbb{Q}_{\ell}\right)$ as $\operatorname{Gal}(K)$-representations, by comparison isomorphisms.

The approach we take for finding this idempotent is motivated by the following well known, elementary lemma on polarized Hodge structures; see [Via13, Lem. 1.6] and [Voi04, Lem. 5]. In the notation of Proposition 1.3, we will apply the lemma with $H=H^{1}\left(C_{\mathbb{C}}, \mathbb{Q}\right), H^{\prime}=H^{2 n+1}\left(X_{\mathbb{C}}, \mathbb{Q}\right)$, and $\gamma=\gamma_{*}$.

Lemma 2.3. Let $(H, Q)$ and $\left(H^{\prime}, Q^{\prime}\right)$ be polarized rational Hodge structures of weight $i$ and let $\gamma: H \rightarrow H^{\prime}$ be a morphism of Hodge structures. Then

$$
H=\operatorname{Im}\left(\gamma^{\prime}\right) \oplus^{\perp_{Q}} \operatorname{Ker}(\gamma) \quad \text { and } \operatorname{Im}\left(\gamma^{\prime}\right)=\operatorname{Im}\left(\gamma^{\prime} \circ \gamma\right)
$$

where $\gamma^{\prime}$ is the transpose of $\gamma$ with respect to the polarizations; i.e., $\gamma^{\prime}=Q \circ \gamma^{\vee} \circ Q^{\prime}$ with $\gamma^{\vee}$ the dual of $\gamma$.

Going forward, we fix the standard principal polarization $Q$ on the rational Hodge structure $H^{1}\left(C_{\mathbb{C}}, \mathbb{Q}\right)$. Recall that this is induced by the cup-product, and that the identification $H^{1}\left(C_{\mathbb{C}}, \mathbb{Q}\right)=$ $H^{1}\left(C_{\mathbb{C}}, \mathbb{Q}\right)^{\vee} \otimes \mathbb{Q}(-1)$ via $Q$ is given by the action of the diagonal $\Delta_{C} \in \mathrm{CH}^{1}(C \times C)_{\mathbb{Q}}$ on $H^{1}\left(C_{\mathbb{C}}, \mathbb{Q}\right)$. Recall also that the dual morphism

$$
\left(\gamma_{*}\right)^{\vee}: H^{2 n+1}\left(X_{\mathbb{C}}, \mathbb{Q}(n)\right)^{\vee} \rightarrow H^{1}\left(C_{\mathbb{C}}, \mathbb{Q}\right)^{\vee}
$$

is identified via Poincaré duality with the morphism of Hodge structures

$$
{ }^{t} \gamma_{*}=\gamma^{*}: H^{2 d-2 n-1}\left(X_{\mathbb{C}}, \mathbb{Q}(d-n)\right) \rightarrow H^{1}\left(C_{\mathbb{C}}, \mathbb{Q}(1)\right),
$$

where ${ }^{t} \gamma$ is the transpose of $\gamma$ in $\mathrm{CH}^{n+1}\left(X \times_{K} C\right)$. We now show that Theorem 2.1 will follow if we can endow $H^{\prime}=H^{2 n+1}\left(X_{\mathbb{C}}, \mathbb{Q}(n)\right)$ with a polarization $Q^{\prime}$ that is induced by a correspondence defined over $K$.

Lemma 2.4. Let $X, C$ and $\gamma$ be as in Proposition 1.3. Let $Q$ be the standard polarization on $H^{1}\left(C_{\mathbb{C}}, \mathbb{Q}\right)$. If there are a polarization $Q^{\prime}$ of $H^{2 n+1}\left(X_{\mathbb{C}}, \mathbb{Q}(n)\right)$ and a correspondence $\Theta \in$ $\mathrm{CH}^{2 d-2 n-1}\left(X \times_{K} X\right)_{\mathbb{Q}}$ making the following diagram commute:

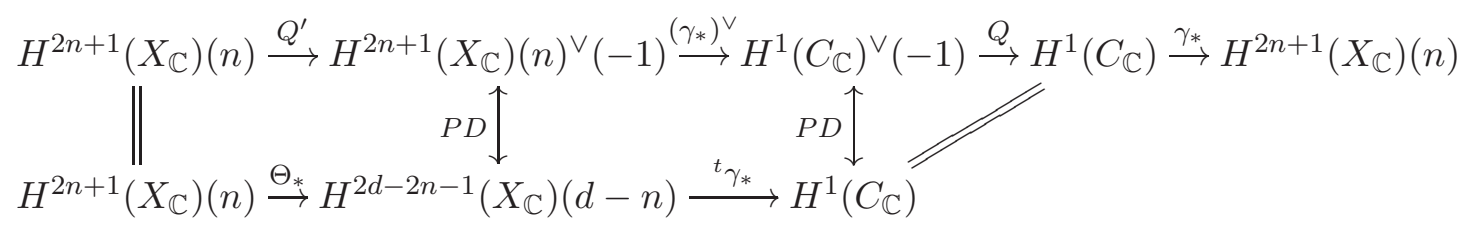

then there exists an abelian subvariety $J \subseteq J_{C / K}$ defined over $K$, and a correspondence defined over $K$, that induces for all prime numbers $\ell$ a split inclusion of $\mathrm{Gal}(K)$-representations

$$
H^{1}\left(J_{\bar{K}}, \mathbb{Q}_{\ell}\right) \hookrightarrow H^{2 n+1}\left(X_{\bar{K}}, \mathbb{Q}_{\ell}(n)\right),
$$

with image $\mathrm{N}^{n} H^{2 n+1}\left(X_{\bar{K}}, \mathbb{Q}_{\ell}(n)\right)$. Moreover, there is a correspondence over $K$ inducing for all prime $\ell$ a splitting for (2.3). 
Proof. As discussed above, up to the statement involving a splitting for (2.3), it is enough to exhibit a correspondence $\pi \in \mathrm{CH}^{1}\left(C \times_{K} C\right)_{\mathbb{Q}}$ which acts idempotently on $H^{1}\left(C_{\mathbb{C}}, \mathbb{Q}\right)$ such that $\pi_{*} H^{1}\left(C_{\mathbb{C}}, \mathbb{Q}\right)$ is mapped via $\gamma_{*}$ isomorphically onto $\operatorname{Im}\left(\gamma_{*}\right)=\mathrm{N}^{n} H^{2 n+1}\left(X_{\mathbb{C}}, \mathbb{Q}(n)\right)$. Following the notation of Lemma 2.3, set $\gamma_{*}^{\prime}=Q \circ\left(\gamma_{*}\right)^{\vee} \circ Q^{\prime}$, and set $p: H^{1}\left(C_{\mathbb{C}}, \mathbb{Q}\right) \rightarrow \operatorname{Im}\left(\gamma_{*}^{\prime}\right)$ to be the orthogonal projection. Consider the endomorphism

$$
\gamma_{*}^{\prime} \circ \gamma_{*}={ }^{t} \gamma_{*} \circ \Theta_{*} \circ \gamma_{*}: H^{1}\left(C_{\mathbb{C}}, \mathbb{Q}\right) \rightarrow H^{1}\left(C_{\mathbb{C}}, \mathbb{Q}\right) .
$$

From Lemma 2.3, the endomorphism $\gamma_{*}^{\prime} \circ \gamma_{*}$ induces an automorphism of $\operatorname{Im}\left(\gamma_{*}^{\prime}\right)$. By the CayleyHamilton theorem, there is a polynomial $P$ with $\mathbb{Q}$-coefficients such that the inverse of $\left.\left(\gamma_{*}^{\prime} \circ \gamma_{*}\right)\right|_{\operatorname{Im}\left(\gamma_{*}^{\prime}\right)}$ is $P\left(\left.\left(\gamma_{*}^{\prime} \circ \gamma_{*}\right)\right|_{\operatorname{Im}\left(\gamma_{*}^{\prime}\right)}\right)$. It is then apparent that $p$ is induced by

$$
\pi:={ }^{t} \gamma \circ \Theta \circ \gamma \circ P\left({ }^{t} \gamma \circ \Theta \circ \gamma\right) \in \mathrm{CH}^{1}\left(C \times{ }_{K} C\right)_{\mathbb{Q}}
$$

since by construction it acts as the identity on $\operatorname{Im}\left(\gamma_{*}^{\prime}\right)$ and as zero on $\operatorname{Ker}\left(\gamma_{*}\right)$. That $\operatorname{Im}(\gamma \circ \pi)_{*}=$ $\operatorname{Im}\left(\gamma_{*}\right)$ follows from Lemma 2.3.

Finally, concerning the splitting for (2.3), writing the correspondence $\pi$ of formula (2.4) as $\pi=\varphi \circ \gamma$, we see that a $\operatorname{Gal}(K)$-equivariant splitting to the inclusion

$$
\gamma_{*}: H^{1}\left(J_{\bar{K}}, \mathbb{Q}_{\ell}\right) \cong \pi_{*} H^{1}\left(C_{\bar{K}}, \mathbb{Q}_{\ell}\right) \hookrightarrow H^{2 n+1}\left(X_{\bar{K}}, \mathbb{Q}_{\ell}(n)\right)
$$

is given by the action $\varphi_{*}$ of $\varphi$.

Up to proving Lemma 2.6, the proof of Theorem 2.1 is now complete.

Remark 2.5. In motivic terms, we have proved the following. With the notations of And04, denote $h^{1}(J)$ the weight-1 direct summand of the homological motive of $J$, and $h(X)$ the homological motive of $X$. We proved that the morphism of motives

$$
h^{1}(J) \cong(C, \pi) \stackrel{\gamma \circ \pi}{\longrightarrow} h(X)(n)
$$

is split injective with a splitting given by the correspondence $\pi \circ \varphi: h(X)(n) \rightarrow(C, \pi)$, and that the cohomology of $\operatorname{Im}(\gamma \circ \pi)$ is $\mathrm{N}^{n} H^{2 n+1}\left(X_{\bar{K}}, \mathbb{Q}_{\ell}(n)\right)$.

Lemma 2.6. Let $X$ be a smooth projective variety of pure dimension $d$ over $K \subseteq \mathbb{C}$. Assume any of (a)-(d) of Theorem [2.1. Then there is a correspondence $\Theta \in \mathrm{CH}^{d-2 n-1}\left(X \times_{K} X\right)$ such that the pairing $(x, y) \mapsto \int_{X_{\mathbb{C}}} x \cup \Theta_{*} y$ defines a polarization $Q^{\prime}$ on $H^{2 n+1}\left(X_{\mathbb{C}}, \mathbb{Q}(n)\right)$; i.e., such that (2.2) is commutative.

Proof. Let us first recall how to polarize the Hodge structure $H^{n}\left(X_{\mathbb{C}}, \mathbb{Q}\right)$ using the Lefschetz decomposition formula, for any smooth projective variety $X$ of pure dimension $d$. We fix the class $L$ of a smooth hyperplane section of $X$. By the Lefschetz hyperplane theorem, the powers of $L$ induce isomorphisms $L^{d-n}: H^{n}\left(X_{\mathbb{C}}, \mathbb{Q}\right) \rightarrow H^{2 d-n}\left(X_{\mathbb{C}}, \mathbb{Q}(d-n)\right)$ for $n \leq d$. By Poincaré duality, the bilinear form $Q_{n}$ on $H^{n}\left(X_{\mathbb{C}}, \mathbb{Q}\right)$ given by

$$
Q_{n}(x, y)=\int_{X_{\mathbb{C}}} x \cup L^{d-n} y
$$

is non-degenerate. By definition, for $n \leq d$, the primitive part of $H^{n}\left(X_{\mathbb{C}}, \mathbb{Q}\right)$ is

$$
H^{n}\left(X_{\mathbb{C}}, \mathbb{Q}\right)_{\text {prim }}:=\operatorname{Ker}\left(L^{d-n+1}: H^{n}\left(X_{\mathbb{C}}, \mathbb{Q}\right) \rightarrow H^{2 d-n+2}\left(X_{\mathbb{C}}, \mathbb{Q}\right)\right) .
$$

We have the Lefschetz decomposition formula

$$
H^{n}\left(X_{\mathbb{C}}, \mathbb{Q}\right)=\bigoplus_{0 \leq r} L^{r} H^{n-2 r}\left(X_{\mathbb{C}}, \mathbb{Q}(-r)\right)_{\text {prim }}
$$


The Hodge index theorem states that decomposition (2.5) is orthogonal for $Q_{n}$ and that the subHodge structure $L^{r} H^{n-2 r}\left(X_{\mathbb{C}}, \mathbb{Q}(-r)\right)_{\text {prim }}$ endowed with the form $(-1)^{r} Q_{n}$ is polarized. We let $p^{n, r}$ denote the orthogonal projector

$$
H^{n}\left(X_{\mathbb{C}}, \mathbb{Q}\right) \rightarrow L^{r} H^{n-2 r}\left(X_{\mathbb{C}}, \mathbb{Q}(-r)\right)_{\text {prim }} \rightarrow H^{n}\left(X_{\mathbb{C}}, \mathbb{Q}\right),
$$

and we define

$$
s_{n}:=\sum_{r}(-1)^{r} p^{n, r} \quad \text { for } n \leq d, \quad \text { and } \quad s_{n}:=\sum_{r}(-1)^{r t} p^{2 d-n, r} \quad \text { for } n>d .
$$

With these notations, the bilinear form $Q^{\prime}$ on $H^{n}\left(X_{\mathbb{C}}, \mathbb{Q}\right)$ given by $Q^{\prime}(x, y)=\int_{X_{\mathbb{C}}} x \cup L^{d-n} s_{n} y$ is a polarization. (Here, $L^{r}$ for $r<0$ is defined to be the inverse of $L^{-r}$.)

Let us now consider the polarized Hodge structure $H^{2 n+1}\left(X_{\mathbb{C}}, \mathbb{Q}(n)\right)$ and proceed to the proof of Lemma 2.6.

(a) If $2 n+1=1$, then note that $H^{1}\left(X_{\mathbb{C}}, \mathbb{Q}\right)=H^{1}\left(X_{\mathbb{C}}, \mathbb{Q}\right)_{\text {prim }}$ so that $s_{1}$ is induced by the diagonal $\Delta_{X} \in \mathrm{CH}^{d}(X \times X)_{\mathbb{Q}}$. Therefore $\Theta=L^{n-1}$ is suitable. (In fact, in the case $n=0$, Theorem 2.1 holds with $J=\operatorname{Pic}_{X}^{0}$.)

If $2 n+1=3$, let us write $\Lambda \in \mathrm{CH}^{1}(X \times X)_{\mathbb{Q}}$ for a correspondence that induces the inverse to the Lefschetz isomorphism $L^{d-1}: H^{1}\left(X_{\mathbb{C}}, \mathbb{Q}\right) \stackrel{\simeq}{\rightarrow} H^{2 d-1}\left(X_{\mathbb{C}}, \mathbb{Q}(d-1)\right.$ ) (Such a correspondence $\Lambda$ does exist; see for instance the proof of Proposition 1.3.) We define

$$
P^{3,1}:=L \circ \Lambda \circ L^{d-2} \in \mathrm{CH}^{d}(X \times X)_{\mathbb{Q}} .
$$

It is clear that $P^{3,1}$ acts as $p^{3,1}$ on $H^{3}\left(X_{\mathbb{C}}, \mathbb{Q}(1)\right)$; we may then define $P^{3,0}=\Delta_{X}-P^{3,1}$, and $S_{3}:=P^{3,0}-P^{3,1} \in \mathrm{CH}^{d}(X \times X)_{\mathbb{Q}}$ acts on $H^{3}\left(X_{\mathbb{C}}, \mathbb{Q}(1)\right)$ as $s_{3}$. Hence $\Theta:=L^{d-2} \circ S_{3}$ is suitable.

(b) If $X$ satisfies the Lefschetz standard conjecture, then Kleiman [Kle68] has shown that the projectors $p^{2 n+1, r}$ are induced by correspondences $P^{2 n+1, r} \in \mathrm{CH}^{d}(X \times X)_{\mathbb{Q}}$. Therefore, denoting $\Lambda \in \mathrm{CH}^{2 d-2 n-1}(X \times X)_{\mathbb{Q}}$ either the correspondence $L^{d-2 n-1}$ if $2 n+1 \leq d$ or a correspondence inducing the inverse of the action of $L^{2 n+1-d}$ on $H^{2 d-2 n-1}\left(X_{\mathbb{C}}, \mathbb{Q}(d-n-1)\right)$ if $2 n+1>d$, we get that $\Theta:=\Lambda \circ\left(\sum_{r}(-1)^{r} P^{2 n+1, r}\right)$ is suitable.

(c) Let $Y$ be a smooth projective threefold, and let $\Gamma \in \mathrm{CH}^{n+2}\left(Y \times_{K} X\right)_{\mathbb{Q}}$ be such that

$$
\Gamma_{*}: H^{3}\left(Y_{\mathbb{C}}, \mathbb{Q}(1)\right) \rightarrow H^{2 n+1}\left(X_{\mathbb{C}}, \mathbb{Q}(n)\right)
$$

is surjective. The polarization on $H^{3}\left(Y_{\mathbb{C}}, \mathbb{Q}(1)\right)$ coming from the Lefschetz decomposition induces a polarization on $H^{2 n+1}\left(X_{\mathbb{C}}, \mathbb{Q}(n)\right)$ and we are going to show that this can be done algebrogeometrically. By (a), there is a correspondence $S_{Y} \in \mathrm{CH}^{3}(Y \times Y)_{\mathbb{Q}}$ such that the pairing $(x, y) \mapsto \int_{Y_{\mathbb{C}}} x \cup\left(S_{Y}\right)_{*} y$ defines a polarization on $H^{3}\left(Y_{\mathbb{C}}, \mathbb{Q}(1)\right)$. By Lemma 2.3, we get a decomposition

$$
H^{3}\left(Y_{\mathbb{C}}, \mathbb{Q}(1)\right)=\operatorname{Im}\left(\left(S_{Y}\right)_{*} \circ \Gamma^{*} \circ L^{d-2 n-1}\right) \oplus \operatorname{Ker}\left(\Gamma_{*}\right) .
$$

It follows that $\Gamma \circ S_{Y} \circ{ }^{t} \Gamma \circ L^{d-2 n-1}$ induces an automorphism of $H^{2 n+1}\left(X_{\mathbb{C}}, \mathbb{Q}(n)\right)$. Consider the correspondence

$$
\Gamma^{\prime}:=S_{Y} \circ{ }^{t} \Gamma \circ L^{d-2 n-1} \in \mathrm{CH}_{n+2}(X \times Y)_{\mathbb{Q}}
$$

The bilinear form

$$
Q^{\prime}(x, y):=\int_{Y_{\mathbb{C}}}\left(\Gamma^{\prime}\right)_{*} x \cup\left(S_{Y}\right)_{*}\left(\Gamma^{\prime}\right)_{*} y=\int_{X_{\mathbb{C}}} x \cup\left(\Gamma^{\prime}\right)^{*}\left(S_{Y}\right)_{*}\left(\Gamma^{\prime}\right)_{*} y
$$

then defines a polarization on $H^{2 n+1}\left(X_{\mathbb{C}}, \mathbb{Q}(n)\right)$. The correspondence $\Theta:={ }^{t} \Gamma^{\prime} \circ S_{Y} \circ \Gamma^{\prime}$ is then suitable.

(d) is a special case of (c) because if $H^{2 n+1}\left(X_{\mathbb{C}}, \mathbb{Q}(n)\right)$ is of geometric coniveau $n$, then by Proposition 1.3, $H^{2 n+1}\left(X_{\mathbb{C}}, \mathbb{Q}(n)\right)$ is of geometric niveau $n$ (meaning that there is a smooth projective curve 
$C$ over $K$ and a correspondence $\gamma \in \mathrm{CH}^{n+1}\left(C \times_{K} X\right)$ such that $\gamma_{*}: H^{1}\left(C_{\mathbb{C}}, \mathbb{Q}\right) \rightarrow H^{2 n+1}\left(X_{\mathbb{C}}, \mathbb{Q}(n)\right)$ is surjective) and hence of geometric niveau $n-1$.

\section{Algebraic Representatives over algebraically Closed fields}

In this section we review Murre's work Mur85] on algebraic representatives over algebraically closed fields. The theory of algebraic representatives is indeed the starting point to showing in Theorem 5.1 that the image of the Abel-Jacobi map $A J: \mathrm{A}^{2}\left(X_{\mathbb{C}}\right) \rightarrow J^{3}\left(X_{\mathbb{C}}\right)$ descends to a field of definition of $X$. In 33.3 , we recall the functorial properties of Chow and Albanese schemes under field extension, and similar results for homomorphisms of abelian varieties. This is used in 33.4 to establish algebraically closed base change for algebraic representatives; see our main Theorem 3.7.

3.1. Preliminaries on Chow groups and algebraic representatives. Fix an algebraically closed field $k$. Recall following Samuel (see [Mur85, Def. 1.6.1] or [Sam60, 2.5]) that given an abelian variety $A$ over $k$, a homomorphism of groups

$$
\mathrm{A}^{i}(X) \stackrel{\phi}{\longrightarrow} A(k)
$$

is said to be regular if for every pair $(T, Z)$ with $T$ a pointed smooth integral variety, and $Z \in$ $\mathrm{CH}^{i}(T \times X)$, the composition

$$
T(k) \stackrel{w_{Z}}{\longrightarrow} \mathrm{A}^{i}(X) \stackrel{\phi}{\longrightarrow} A(k)
$$

is induced by a morphism of varieties $\psi_{Z}: T \rightarrow A$ over $k$, where, if $t_{0} \in T(k)$ is the base point of $T, w_{Z}: T(k) \rightarrow \mathrm{A}^{i}(X)$ is given by $t \mapsto Z_{t}-Z_{t_{0}}$; here $Z_{t}$ is the refined Gysin fiber (see e.g., Ful98, Ch.10]).

Given a surjective regular homomorphism $\phi: \mathrm{A}^{i}(X) \rightarrow A(k)$, there exists a correspondence $Z \in \mathrm{CH}^{i}(A \times X)$ such that $\phi \circ w_{Z}$ is an isogeny (e.g., Mur85, Cor. 1.6.3] ; see also Lemma 4.9).

Definition 3.1. An algebraic representative for $\mathrm{A}^{i}(X)$ is an abelian variety that is initial for regular homomorphisms. In other words, it is a pair $\left(\mathrm{Ab}^{i}(X), \phi_{X}^{i}\right)$ with $\mathrm{Ab}^{i}(X)$ an abelian variety over $k$, and

$$
\mathrm{A}^{i}(X) \stackrel{\phi_{X}^{i}}{\longrightarrow} \mathrm{Ab}^{i}(X)(k)
$$

a regular homomorphism of groups such that for every pair $(A, \phi)$ consisting of an abelian variety $A$ over $k$ and a regular homomorphism of groups $\phi: \mathrm{A}^{i}(X) \rightarrow A(k)$, there exists a unique morphism $f: \operatorname{Ab}^{i}(X) \rightarrow A$ of varieties over $k$ such that the following diagram commutes

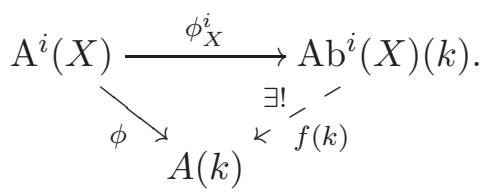

Algebraic representatives, if they exist, are unique up to unique isomorphism. For an algebraic representative, the homomorphism $\phi_{X}^{i}$ is surjective (e.g., [Mur85, Rem. a), p.225]). It is well known that algebraic representatives of $\mathrm{A}^{1}(X)$ and $\mathrm{A}^{d}(X)$ (where $d$ is the dimension of $X$ ) do exist : the Abel-Jacobi map taking a divisor to its associated line bundle $A^{1}(X) \rightarrow \operatorname{Pic}^{0}(X)(k)$ and the Albanese map $A^{d}(X) \rightarrow \operatorname{Alb}(X)(k)$ are universal surjective regular homomorphisms.

We will frequently use the following necessary and sufficient condition for the existence of an algebraic representative. 
Proposition 3.2 ([Sai79, Thm. 2.2], Mur85, Prop. 2.1]). An algebraic representative for $\mathrm{A}^{i}(X)$ exists if and only if there exists a constant $M$ such that for every surjective regular homomorphism $\phi: \mathrm{A}^{i}(X) \rightarrow A(k)$ we have $\operatorname{dim}_{k} A \leq M$.

Remark 3.3. Note that the notions of regular homomorphism and of algebraic representative are usually defined for smooth projective irreducible varieties over an algebraically closed field $k$. However it is harmless to consider possibly disconnected smooth projective varieties: for instance, a smooth projective variety $X$ over $k$ admits an algebraic representative $\left(\operatorname{Ab}^{i}(X), \phi_{X}^{i}\right)$ for $\mathrm{A}^{i}(X)$ if and only if each of its irreducible components $X_{1}, \ldots, X_{n}$ admits an algebraic representative $\left(\operatorname{Ab}^{i}\left(X_{j}\right), \phi_{X_{j}}^{i}\right)$ for $\mathrm{A}^{i}\left(X_{j}\right), j=1, \ldots, n$. In this case, $\left(\mathrm{Ab}^{i}(X), \phi_{X}^{i}\right) \cong \bigoplus_{j=1}^{n}\left(\operatorname{Ab}^{i}\left(X_{j}\right), \phi_{X_{j}}^{i}\right)$.

3.2. Results of Murre on codimension two cycles. We recall Murre's main result concerning algebraic representatives for codimension two cycles.

Theorem 3.4 ([Mur85, Thm. A, p.226, Thm. C, p.229, Thm. 10.3, p.258]). Let $X$ be a smooth projective variety over an algebraically closed field $k$.

(a) There exists an algebraic representative $\left(\mathrm{Ab}^{2}(X), \phi_{X}^{2}\right)$ of $\mathrm{A}^{2}(X)$.

(b) For all but finitely many primes $\ell \neq$ char $k$, there is a natural inclusion

$$
\left.H^{1}\left(\widehat{\mathrm{Ab}^{2}(X}\right), \mathbb{Z}_{\ell}\right) \hookrightarrow H^{3}\left(X, \mathbb{Z}_{\ell}(1)\right) .
$$

(c) For all primes $\ell \neq$ char $k$, there is a natural inclusion

$$
\left.H^{1}\left(\widehat{\mathrm{Ab}^{2}(X}\right), \mathbb{Q}_{\ell}\right) \hookrightarrow H^{3}\left(X, \mathbb{Q}_{\ell}(1)\right) .
$$

(d) If $k=\mathbb{C}$, then an algebraic representative is given by the image of the Abel-Jacobi map. In other words, the pair $\left(J_{\mathrm{a}}^{3}(X), \psi^{2}\right)$, where

$$
\psi^{2}: \mathrm{A}^{2}(X) \rightarrow J^{3}(X)
$$

is the Abel-Jacobi map, and $J_{\mathrm{a}}^{3}(X)=\operatorname{Im}\left(\psi^{2}\right)$, is an algebraic representative of $\mathrm{A}^{2}(X)$. For each $\ell$, the Abel-Jacobi map $\psi^{2}$ is an injection on torsion, so that there is an isomorphism:

$$
T_{\ell} \mathrm{A}^{2}(X) \stackrel{T_{\ell} \psi^{2}}{\cong} T_{\ell} J_{\mathrm{a}}^{3}(X) .
$$

Remark 3.5. The inclusions (3.1) and (3.2) are not stated explicitly in Mur85; the argument is contained in Murre's argument that the second Bloch map is injective [Mur85, Prop. 9.2, p.256]. More precisely, [Mur85, (18), p.250] establishes that for almost all $\ell$ there is an inclusion

$$
\left.H^{1}\left(\widehat{\mathrm{Ab}^{2}(X}\right), \mathbb{Z}_{\ell}(1)\right)=H^{1}\left(\operatorname{Ab}^{2}(X), \mathbb{Z}_{\ell}\right)^{\vee}=T_{\ell} \operatorname{Ab}^{2}(X) \subseteq T_{\ell} \mathrm{A}^{2}(X) \subseteq T_{\ell} \operatorname{CH}^{2}(X) .
$$

(For the finitely many exceptional $\ell \neq$ char $k$, Murre's argument shows that there is still an inclusion of rational Tate modules $\left.H^{1}\left(\widehat{\mathrm{Ab}^{2}(X}\right), \mathbb{Q}_{\ell}(1)\right)=V_{\ell} \mathrm{Ab}^{2}(X) \subseteq V_{\ell} \mathrm{CH}^{2}(X)$.) Then, Murre's argument that the second Bloch map is injective shows that for almost all $\ell$ there is also an inclusion:

$$
T_{\ell} \mathrm{CH}^{2}(X) \hookrightarrow H^{3}\left(X, \mathbb{Z}_{\ell}(2)\right) .
$$

(This may fail to be an inclusion at the primes for which $H^{3}\left(X, \mathbb{Z}_{\ell}(2)\right)$ has torsion; for cohomology with $\mathbb{Q}_{\ell}$-coefficients, this does not pose a problem, and one obtains the inclusion for all $\ell$.) The inclusion (3.5) is not explicitly stated in [Mur85 but follows immediately from [Mur85, (19)] and [Blo79, Lem. 2.4], together with the fact that $H^{3}\left(X, \mathbb{Z}_{\ell}(2)\right)$ is torsion-free for almost all $\ell$ by a theorem of Gabber Gab83. 
Remark 3.6. We can be more precise about the primes $\ell$ for which the argument above does not establish the inclusion (3.1). By [Mur85, Lem. 1.6.2, p.224] there exists a cycle $Z$ on $X \times \mathrm{Ab}^{2}(X)$ such that the composition $\psi_{Z}:=\phi_{X} \circ w_{Z}: \mathrm{Ab}^{2}(X) \rightarrow \mathrm{A}^{2}(X) \rightarrow \mathrm{Ab}^{2}(X)$ is an isogeny. For each $\ell \nmid \operatorname{deg}\left(\psi_{Z}\right)$, Murre [Mur85, p.250] establishes (3.4). Therefore, (3.1) holds for all primes $\ell$ such that $\ell \nmid \operatorname{deg}\left(\psi_{Z}\right)$ and such that $H^{3}\left(X, \mathbb{Z}_{\ell}(2)\right)$ is torsion-free. Over $\mathbb{C}$, a result of Voisin Voi13, Thm. 1.12, 1.13], Voi15, Thm. 1.7] establishes that for threefolds, if the diagonal admits an integral cohomological decomposition, then there exists a cycle $Z$ so that $\psi_{Z}$ is of degree 1 (see also Voi13, Rem. 1.9]).

3.3. Algebraic representatives, Chow varieties, and traces for primary field extensions. If an algebraic representative exists, then by definition it is dominated by the Albanese varieties of components of the Chow scheme. Since Albanese varieties and Chow schemes respect base change of fields, this gives an approach for studying the problem of base change for algebraic representatives. A key tool for studying base change of abelian varieties for primary extensions of fields is the socalled $L / K$-trace. We review in this section some of the results in the literature, and how we will employ them in the context of algebraic representatives.

3.3.1. Chow rigidity and $L / K$-trace for primary field extensions. We exploit (Chow) rigidity and $L / K$-traces for abelian varieties and primary field extensions. See Con06 for a modern treatment; here, we summarize what we need. Let $L / K$ be a primary extension of fields, i.e., an extension such that the algebraic closure of $K$ in $L$ is purely inseparable over $K$. If $L / K$ is primary and separable (e.g., if $K$ is algebraically closed), then $L / K$ is said to be regular.

If $A$ is an abelian variety over $L$, an $L / K$-trace is a final object $\left(\operatorname{tr}_{L / K}(A), \tau_{A}\right)$ in the category of pairs $(\underline{B}, f)$ where $\underline{B}$ is an abelian variety over $K$ and $f: \underline{B}_{L} \rightarrow A$ is a map of abelian varieties over $L$. An $L / K$-trace exists [Con06, Thm. 6.2], and is unique up to unique isomorphism. For brevity, we will frequently denote the $L / K$-trace by the pair $(\underline{\underline{A}}, \tau)$.

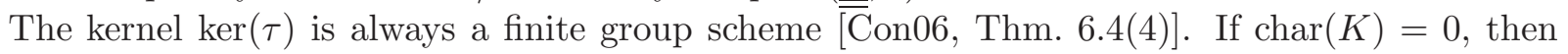
$\operatorname{ker}(\tau)$ is trivial [Con06, p.72]; more generally, if $L / K$ is regular, then $\operatorname{ker}(\tau)$ is a connected group scheme with connected dual [Con06, Thm. 6.4, Thm. 6.12], and thus $\tau$ is purely inseparable. In particular [Con06, p.76], if $L / K$ is regular, then on $L$-points $\tau$ induces an injection

$$
\underline{\underline{A}}(L) \stackrel{\tau(L)}{\longrightarrow} A(L) .
$$

The image of $\tau$ in $A$ descends to $K$ if and only if $\operatorname{Ker}(\tau)$ is the trivial group scheme Con06, p.72]. As we have seen, this is automatic in characteristic zero, but need not hold in positive characteristic.

Chow's rigidity theorem (see [Con06, Thm. 3.19]) states that if $\underline{B}$ and $\underline{C}$ are both abelian varieties over $K$ (and $L / K$ is still primary), then the natural inclusion

$$
\operatorname{Hom}_{K}(\underline{B}, \underline{C}) \rightarrow \operatorname{Hom}_{L}\left(\underline{B}_{L}, \underline{C}_{L}\right)
$$

is a bijection. In particular, if $A / L$ and $\underline{B} / K$ are abelian varieties, then there are canonical bijections

$$
\operatorname{Hom}_{L}\left(\underline{B}_{L}, A\right)=\operatorname{Hom}_{L}\left(\underline{B}_{L}, \underline{\underline{A}}_{L}\right)=\operatorname{Hom}_{K}(\underline{B}, \underline{\underline{A}}),
$$

where the first equality is the universal property of the $L / K$-trace, and the second equality comes from rigidity.

3.3.2. Chow varieties, Albaneses, and algebraic representatives. If $X / K$ is a projective variety over a perfect field $K$, then for each $i$ there is a Chow scheme $\operatorname{Chow}^{i}(X)$ over $K$, a union of countably many connected components, whose points parametrize cycles of codimension $i$. If $L / K$ is an 
extension of fields and $X_{L}:=X_{K} \times_{K} L$, then $\operatorname{Chow}^{i}\left(X_{L}\right) \cong \operatorname{Chow}^{i}(X)_{L}:=\operatorname{Chow}^{i}(X) \times_{K} L$ Fri91, Prop. 1.1] ; see also Kol96.

If $(Y, y) / K$ is a pointed geometrically integral variety over a perfect field, an Albanese variety for $Y$ is an abelian variety $\operatorname{Alb}(Y)$ over $K$ equipped with a pointed map $(Y, y) \rightarrow(\operatorname{Alb}(Y), 0)$ that is initial with respect to pointed maps from $(Y, y)$ to abelian varieties. If $Y / K$ is smooth and proper, then $\operatorname{Alb}(Y)=\operatorname{Pic}_{\left(\operatorname{Pic}_{Y / K}^{0}\right)_{\text {red } / K}}^{0}$; since the Picard functor behaves well under base change, we have $\operatorname{Alb}\left(Y_{L}\right) \cong \operatorname{Alb}(Y) \times_{K} L$ for any extension of perfect fields $L / K$. For geometrically integral varieties $Y / K$ that are not smooth and proper, either using (a compactification and) resolution of singularities in characteristic 0, or an argument via de Jong's alterations as in [Moc12, Cor. A.11], one may again show that $Y$ admits an Albanese, and that $\operatorname{Alb}\left(Y_{L}\right) \cong \operatorname{Alb}(Y) \times_{K} L$.

One setting where we will use Albaneses extensively (e.g., the proof of Theorem 13.7) is the following. Let $X$ be a smooth projective variety defined over an algebraically closed field $k$, and assume there is an algebraic representative $\left(\mathrm{Ab}^{i}(X), \phi_{X}^{i}\right)$. From the discussion above, we see that $\mathrm{Ab}^{i}(X)$ is the colimit of the inverse system of abelian varieties obtained from the Albanese varieties of components of the Chow scheme $\mathrm{Chow}^{i}(X)$. In fact, one should work with Albaneses of smooth alterations of the reduction of products of components of the Hilbert scheme, but we avoid this technical point in this discussion. In particular, $\mathrm{Ab}^{i}(X)$ admits a surjection from a finite product of these abelian varieties. Moreover, if $K \subseteq k$ is a perfect field, and $\underline{X}$ is defined over $K$ with $X=\underline{X} \times_{K} k$, then $\operatorname{Ab}^{i}(X)$ admits a morphism from the pullback to $k$ of the inverse system of torsors under abelian varieties defined over $K$ obtained from the Albanese torsors of components of the Chow scheme $\operatorname{Chow}^{i}(\underline{X})$. In particular, since every component of $\operatorname{Chow}^{i}(X)$ is a component of a pullback to $k$ of a component of $\operatorname{Chow}^{i}(\underline{X})$, we have that $\operatorname{Ab}^{i}(X)$ admits a surjection from a torsor under an abelian variety defined over $K$. (In fact, it will follow from Theorems 3.7 and 4.4 that if $k=\bar{K}$, or $\operatorname{char}(k)=0$, then $\mathrm{Ab}^{i}(X)$, if it exists, descends to an abelian variety over $K$.)

3.4. Algebraically closed base change for algebraic representatives. The aim of this subsection is to establish our main addition to Murre's work on algebraic representatives over algebraically closed fields :

Theorem 3.7. Let $\Omega / k$ be an extension of algebraically closed fields. Let $X / k$ be a smooth projective variety. Assume that either $\mathrm{A}^{i}(X)$ or $\mathrm{A}^{i}\left(X_{\Omega}\right)$ admits an algebraic representative. Then both $\mathrm{A}^{i}(X)$ and $\mathrm{A}^{i}\left(X_{\Omega}\right)$ admit an algebraic representative.

Moreover, let $\left(\mathrm{Ab}^{i}(X), \phi_{X}^{i}\right)$ and $\left(\mathrm{Ab}^{i}\left(X_{\Omega}\right), \phi_{X_{\Omega}}^{i}\right)$ be algebraic representatives for $\mathrm{A}^{i}(X)$ and $\mathrm{A}^{i}\left(X_{\Omega}\right)$, respectively. Then there are natural maps

$$
\mathrm{Ab}^{i}(X) \stackrel{b^{\prime}}{\longrightarrow}{\underline{\underline{\mathrm{Ab}^{i}}}}^{i}\left(X_{\Omega}\right) \text { and }{\underline{\underline{\mathrm{Ab}^{i}}}}^{(}\left(X_{\Omega}\right)_{\Omega} \stackrel{\tau}{\longrightarrow} \mathrm{Ab}^{i}\left(X_{\Omega}\right)
$$

over $k$ and $\Omega$, respectively, where $\left(\underline{\underline{A b}}^{i}\left(X_{\Omega}\right), \tau\right)$ is the $\Omega / k$-trace of $\mathrm{Ab}^{i}\left(X_{\Omega}\right)$ (3.3.1). In characteristic zero, $b^{\prime}$ and $\tau$ are isomorphisms; in positive characteristic, they are purely inseparable isogenies. In particular, in characteristic zero, there is a natural isomorphism $\operatorname{Ab}^{i}(X)_{\Omega} \cong \operatorname{Ab}^{i}\left(X_{\Omega}\right)$.

We start by recalling a rigidity result for torsion in Chow groups.

Theorem 3.8 (Lecomte Lec86, Thm. 3.11]). Let $\Omega / k$ be an extension of algebraically closed fields, and let $X$ be a projective variety over $k$. Base change on cycles induces an injective homomorphism of Chow groups $b_{\Omega / k}: \mathrm{CH}^{i}(X) \rightarrow \mathrm{CH}^{i}\left(X_{\Omega}\right)$. For each positive integer $N, b_{\Omega / k}$ induces isomorphisms on the $N$-torsion groups:

(a) $b_{\Omega / k}[N]: \mathrm{CH}^{i}(X)[N] \stackrel{\sim}{\longrightarrow} \mathrm{CH}^{i}\left(X_{\Omega}\right)[N]$;

(b) $b_{\Omega / k}[N]: \mathrm{A}^{i}(X)[N] \stackrel{\sim}{\longrightarrow} \mathrm{A}^{i}\left(X_{\Omega}\right)[N]$. 
Proof. That the base-change homomorphism $b_{\Omega / k}: \mathrm{CH}^{i}(X) \rightarrow \mathrm{CH}^{i}\left(X_{\Omega}\right)$ is injective is standard and is proved using a spreading out argument followed by a specialization argument (e.g., the argument of [Blo10, Lem. 1A.3, p.22]). Precisely, denoting $r_{\Omega / k}: \mathrm{CH}^{i}\left(X_{\Omega}\right) \rightarrow \mathrm{CH}^{i}(X)$ the specialization homomorphism (see [Blo10, Lem. 1A.3, p.22] and [Ful98, §20.3]), we have that $r_{\Omega / k} \circ b_{\Omega / k}$ is the identity on $\mathrm{CH}^{i}(X)$, giving the injectivity of $b_{\Omega / k}$. Part (a) is the content of [Lec86, Thm. 3.11]. As a consequence of (a), $r_{\Omega / k}$ and $b_{\Omega / k}$ are in fact inverses of each other on $N$-torsion. Part (b) then follows from the fact that $b_{\Omega / k}$ and $r_{\Omega / k}$ respect algebraic equivalence.

Proof of Theorem 3.7. We proceed in four steps.

Step 1. Let $X$ be a smooth projective variety defined over $k$, let $A / k$ be an abelian variety, and let $\phi: \mathrm{A}^{i}(X) \rightarrow A(k)$ be a regular homomorphism. Then there is a regular homomorphism $\phi_{\Omega}$ : $\mathrm{A}^{i}\left(X_{\Omega}\right) \rightarrow A_{\Omega}(\Omega)$, which is surjective if $\phi$ is surjective, making the following diagram commute:

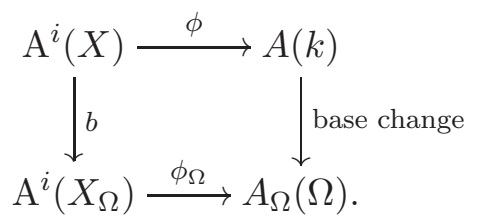

Note that by the criterion of [Sai79, Thm. 2.2], Mur85, Prop. 2.1] (see Proposition 3.2), it follows from Step 1 that if $\mathrm{A}^{i}\left(X_{\Omega}\right)$ has an algebraic representative, then so does $\mathrm{A}^{i}(X)$.

To establish Step 1, we first construct $\phi_{\Omega}$; we thank the referee for providing such a construction. Denote by $\mathrm{A}_{\natural}^{i}(X)$ the contravariant functor on the category of smooth integral varieties over $k$ given by families of algebraically trivial cycles on $X$. More precisely, the functor $\mathrm{A}_{\natural}^{i}(X)$ sends a variety $T$ as above to the group of cycles $Z \in \mathrm{CH}^{i}\left(X \times_{k} T\right)$ such that for some (equivalently, for any) geometric point $x: \operatorname{Spec}\left(\Omega^{\prime}\right) \rightarrow T$ with $\Omega^{\prime}$ being an algebraically closed field over $k$, we have that the cycle $x^{!}(Z) \in \mathrm{CH}^{i}\left(X_{\Omega^{\prime}}\right)$ is algebraically trivial. By definition of a regular homomorphism, we have a morphism of contravariant functors

$$
\phi_{\natural}: \mathrm{A}_{\natural}^{i}(X) \rightarrow A
$$

on the category of smooth integral varieties over $k$. Moreover, both functors $\mathrm{A}_{\natural}^{i}(X)$ and $A$ send inverse limits of varieties to direct limits of abelian groups. Therefore, the morphism of functors $\phi_{\natural}$ extends in a canonical way to the category of schemes over $k$ that can be obtained as inverse limits of smooth integral varieties over $k$. In particular, evaluating on $\operatorname{Spec} \Omega$, we obtain the desired regular homomorphism

$$
\mathrm{A}^{i}\left(X_{\Omega}\right)=\mathrm{A}_{\natural}^{i}(X)(\Omega) \stackrel{\phi_{\natural}(\Omega)}{\longrightarrow} A(\Omega),
$$

which we denote by $\phi_{\Omega}$. Note that this homomorphism is surjective when $\phi$ is surjective due to the existence of the cycle and isogeny from [Mur85, Cor. 1.6.3] (see Lemma 4.9(c)).

Step 2. Let $X$ be a smooth projective variety defined over $k$, let $A / \Omega$ be an abelian variety, and let $\phi_{X_{\Omega}}: \mathrm{A}^{i}\left(X_{\Omega}\right) \rightarrow A(\Omega)$ be a regular homomorphism. Then there is a regular homomorphism $\phi: \mathrm{A}^{i}(X) \rightarrow \underline{\underline{A}}(k)$ to the $k$-points of the $\Omega / k$-trace of $A$ (see 3 3.3.1), making the following diagram commute:

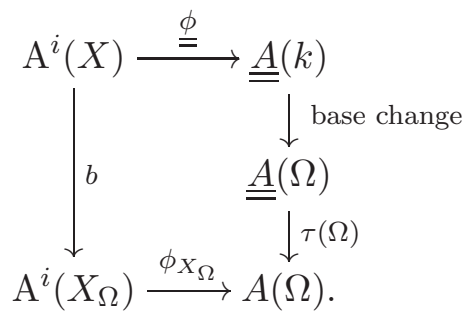


Here, $b=b_{\Omega / k}: \mathrm{A}^{i}(X) \rightarrow \mathrm{A}^{i}\left(X_{\Omega}\right)$ is the base change map on cycles, and $\tau: \underline{\underline{A}}_{\Omega} \rightarrow A$ is the canonical map (see 3.3.1) ; recall that $\tau$ is an isomorphism onto its image in characteristic zero, and is at worst a purely inseparable isogeny onto its image in positive characteristic. In particular, $\tau$ induces an inclusion on $\Omega$-points (3.6).

To establish Step 2, let us define $\phi$. For this, assume that $\alpha \in \mathrm{A}^{i}(X)$. Then there is a smooth projective irreducible curve $T$ over $\overline{\bar{k}}$, with $k$-points $t_{1}, t_{0}$, and a cycle $Z \in \mathrm{CH}^{i}\left(T \times_{k} X\right)$ such that $\alpha=w_{Z, t_{0}}\left(t_{1}\right)$ (e.g., [Ful98, Exa. 10.3.2]). Since $\phi_{X_{\Omega}}$ is regular, there is an $\Omega$-morphism $\gamma: T_{\Omega} \rightarrow A$ that induces $\phi_{X_{\Omega}} \circ w_{Z_{\Omega}}$. The morphism $\gamma$ necessarily factors through the Albanese map $a_{\Omega}: T_{\Omega} \rightarrow \operatorname{Alb}\left(T_{\Omega}\right)$, via a morphism say $\delta: \operatorname{Alb}\left(T_{\Omega}\right) \rightarrow A$. Since $\left(\operatorname{Alb}\left(T_{\Omega}\right), a_{\Omega}\right) \cong(\operatorname{Alb}(T), a)_{\Omega}$, the definition of the $\Omega / k$-trace and rigidity (see 3.3.1) together imply there is a $k$-morphism $\epsilon: \operatorname{Alb}(T) \rightarrow \underline{\underline{A}}$ so that $\delta=\tau \circ \epsilon_{\Omega}$. In other words, there is a commutative diagram

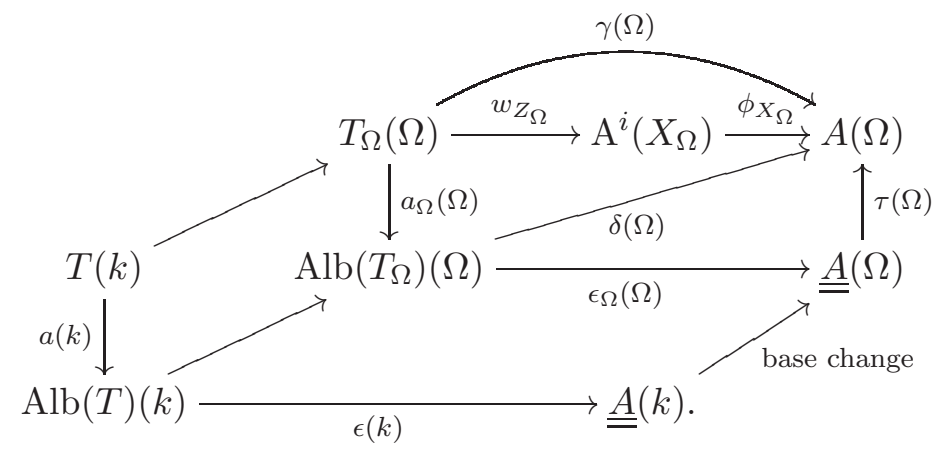

We define $\underline{\phi}(\alpha):=\epsilon\left(a\left(t_{1}\right)-a\left(t_{0}\right)\right)$. Since the base change map on points is injective and since $\operatorname{Ker}(\tau)(\Omega)$ is trivial (see (3.6) $), \underline{\phi}(\alpha)$ is well-defined, i.e., it is independent of the choice of $T$. It is clear from our construction of $\underline{\underline{\phi}}$ that (3.9) commutes. In fact, starting with a general smooth pointed connected variety $T$, the same argument shows that $\underline{\underline{\phi}}$ is regular (since $\epsilon \circ a$ is defined over $k)$.

Step 3. In the notation of Step 2, if $\phi_{X_{\Omega}}$ is surjective, then $\underline{\underline{\phi}}: A^{i}(X) \rightarrow \underline{\underline{A}}(k)$ is surjective and $\tau: \underline{\underline{A}}_{\Omega} \rightarrow A$ is a purely inseparable isogeny (and an isomorphism in characteristic 0 ).

Note that by the criterion of [Sai79, Thm. 2.2], [Mur85, Prop. 2.1] (see Proposition [3.2), it follows from Step 3 and (3.9) that if $\mathrm{A}^{i}(X)$ has an algebraic representative, then so does $\mathrm{A}^{i}\left(X_{\Omega}\right)$.

To establish Step 3, recall that if $\phi_{X_{\Omega}}$ is surjective, then $A / \Omega$ is dominated by an inverse system of abelian varieties defined over $k$ (see the discussion in 3.3 .2 ); in fact, there is an abelian variety $B / k$ (namely the Albanese of a finite union of smooth alterations of components of the Chow scheme) such that there is a surjection $\alpha: B_{\Omega} \rightarrow A$. It follows (see 33.3 .1 ) that $\tau$ is a purely inseparable isogeny (and an isomorphism in characteristic 0 ).

Let us now show that $\underline{\phi}$ is surjective provided $\phi_{X_{\Omega}}$ is surjective. Let $\ell \in \mathbb{N}$ be a prime $\neq$ $\operatorname{char}(k)$, and let us consider $\ell$-torsion in the commutative diagram (3.9). The morphism $\tau$ induces an isomorphism (3.6) $T_{\ell} \tau: T_{\ell} \underline{\underline{A}}(\Omega) \rightarrow T_{\ell} A(\Omega)$. Lecomte's rigidity Theorem 3.8 says that the homomorphism $T_{\ell} b$ is an isomorphism. The base change map $T_{\ell} \underline{\underline{A}}(k) \rightarrow T_{\ell} \underline{\underline{A}}(\Omega)$ on torsion is also an isomorphism. Therefore, a diagram chase in (3.9) shows the surjectivity of $T_{\ell} \underline{\underline{\phi}}$, and thus that of $\underline{\underline{\phi}}$ (Mur85, Lem. 1.6.2(i)]; see Lemma 4.9(a)).

Step 4. Let $\left(\mathrm{Ab}^{i}(X), \phi_{X}^{i}\right)$ and $\left(\mathrm{Ab}^{i}\left(X_{\Omega}\right), \phi_{X_{\Omega}}^{i}\right)$ be algebraic representatives for $\mathrm{A}^{i}(X)$ and $\mathrm{A}^{i}\left(X_{\Omega}\right)$, respectively. The $k$-homomorphism $b^{\prime}: \mathrm{Ab}^{i}(X) \rightarrow{\underline{\underline{\mathrm{Ab}^{i}}}}^{i}\left(X_{\Omega}\right)$ induced by the regular homomorphism $\underline{\underline{\phi}}: A^{i}(X) \rightarrow \underline{\underline{\mathrm{Ab}}}^{i}\left(X_{\Omega}\right)(k)$ of Step 2 is an isomorphism in characteristic zero, and a purely insepa$\overline{\bar{r}}$ able isogeny in positive characteristic. 
It suffices to show that $b^{\prime}(k)$ is an isomorphism. We have the following commutative diagram:

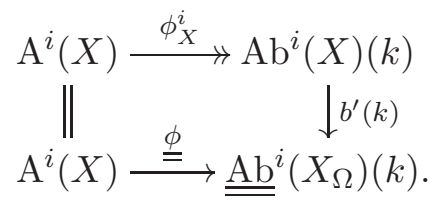

We already know from Step 3 that $\underline{\underline{\phi}}$ is surjective. Therefore, it remains only to prove that $b^{\prime}$ is injective on $k$-points. Let $\zeta \in \overline{\operatorname{Ker}} b^{\prime}(k)$ and let $z \in \mathrm{A}^{i}(X)$ be a cycle mapping to $\zeta$ (i.e., $\phi_{X}^{i}(z)=\zeta$ ). Then $z_{\Omega}:=b(z) \in \mathrm{A}^{i}\left(X_{\Omega}\right)$ is abelian equivalent to 0 (i.e., $z_{\Omega}$ is sent to 0 under any regular morphism), since $\phi_{X_{\Omega}}^{i}\left(z_{\Omega}\right)=0$ by the commutativity of (3.9) and (3.10). In particular, considering the regular homomorphism $\left(\phi_{X}^{i}\right)_{\Omega}$ of Step 1 , we have $\left(\phi_{X}^{i}\right)_{\Omega}\left(z_{\Omega}\right)=0$. By virtue of the commutativity of (3.8), and the injectivity of the base change map, we have that $\phi_{X}^{i}(z)=0$; i.e., $\zeta=0$, and we have that $b^{\prime}$ is injective on geometric points.

\section{Algebraic representatives and Galois actions}

In this section we extend the notion of algebraic representative to the setting of Galois field extensions. The main result of the section is Theorem 4.4, which says that algebraic representatives descend from the algebraic closure of a perfect field. We also establish several basic results on regular homomorphisms in this setting following Mur85.

Throughout this section, we take $k$ to be an algebraically closed field, and $K \subseteq k$ to be a perfect sub-field such that $k / K$ is algebraic. (In particular, $K$ is the fixed field of $\operatorname{Gal}(k / K)$.)

4.1. Preliminaries on Galois descent. If $X / k$ is a variety and $\sigma \in \operatorname{Gal}(k / K)$, let $X^{\sigma}$ be the fiber product $X^{\sigma}=X \times_{\operatorname{Spec} k, \sigma}$ Spec $k$; by definition, it comes equipped with a map $\tilde{\sigma}: X^{\sigma} \rightarrow X$ over $\sigma$. A $k / K$ descent datum on $X$ is a system of isomorphisms $\left\{\sigma_{X}: X \stackrel{\sim}{\rightarrow} X\right.$ over $\left.\sigma: \sigma \in \operatorname{Gal}(k / K)\right\}$ satisfying the co-cycle condition $(\sigma \tau)_{X}=\sigma_{X} \circ \tau_{X}: X \rightarrow X$. There is a similar notion of a descent datum on a morphism of varieties (i.e., a descent datum on its graph), and the category of quasi-projective $k$-schemes equipped with $k / K$ descent data is equivalent to the category of quasi-projective schemes over $K$ (e.g., [BLR90, Thm. 6, p.135, p.141], GW10, Thm. 14.84, p.457]). We will often denote the effect of this functor by $(X,\{\sigma\}) \mapsto \underline{X}$; the inverse functor is $\underline{Y} \mapsto Y:=$ $\underline{Y} \times{ }_{\text {Spec } K}$ Spec $k$. Similar notation will be employed for cycles.

Note that a $k / K$ descent datum on $X$ (or, equivalently, a choice of $K$-model $\underline{X}$ ) determines an action of $\operatorname{Gal}(k / K)$ on the $k$-points $X(k)$; a morphism $X \rightarrow Y$ of $k$-varieties with descent data descends to $K$ if and only if its action on $k$-points is $\operatorname{Gal}(k / K)$-equivariant (e.g., [Mil14, Prop. 16.9]).

4.2. Action of the Galois group on cycles. Let $X / k$ be a quasi-projective variety equipped with a $k / K$-descent datum $\left\{\sigma_{X}\right\}$. Pullback by the flat morphism of schemes $\sigma_{X}$ induces [Ful98, $\S 1.7]$ a homomorphism

$$
\sigma_{X}^{*}: \mathrm{CH}^{i}(X) \rightarrow \mathrm{CH}^{i}(X)
$$

and we have $\sigma_{X}^{*}[Z]=\left[\sigma_{X}^{*} Z\right]$. The co-cycle condition for descent data then yields an action of $\operatorname{Gal}(k / K)$ on $\mathrm{CH}^{i}(X)$, which restricts to an action on $\mathrm{A}^{i}(X)$.

Now let $T / k$ be a smooth, connected variety over $k$; let $Z$ be a codimension $i$ cycle on $T \times_{k} X$, and let $t_{0}:$ Spec $k \rightarrow T$ be a $k$-point. If $T, Z$ and $t_{0}$ are equipped with compatible $k / K$ descent 
data, we obtain a commutative diagram

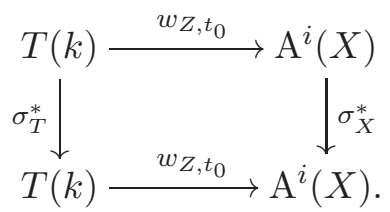

and we see that $w_{Z}$ is $\operatorname{Gal}(k / K)$-equivariant.

Even in the absence of a distinguished $K$-model for $T$ and $Z$ one can show, using the refined Gysin map [Ful98, §6.3], that

$$
\sigma_{X}^{*} Z_{t_{0}}=\left(\left(\tilde{\sigma} \times_{\sigma} \sigma_{X}\right)^{*} Z\right)_{\tilde{\sigma}^{*} t_{0}}
$$

and we thus have a commutative diagram

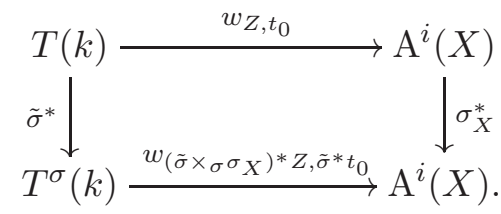

4.3. Galois equivariant algebraic representatives. Let $k$ be an algebraically closed field, and $K \subseteq k$ a perfect sub-field such that $k / K$ is algebraic. Let $X$ be a smooth projective variety over $k$, equipped with $k / K$ descent data. Let $A$ be an abelian variety over $k$ that is also equipped with $k / K$ descent data. We say that a regular homomorphism

$$
\phi: \mathrm{A}^{i}(X) \rightarrow A(k)
$$

is $\operatorname{Gal}(k / K)$-equivariant if for each $\sigma \in \operatorname{Gal}(k / K)$, the following diagram is commutative:

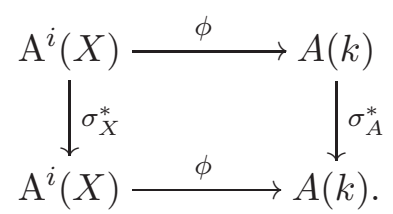

Remark 4.1. Given such data $\left(A,\left\{\sigma_{A}\right\}, \phi\right)$, the class $[0] \in \mathrm{A}^{i}(X)$ is fixed by $\operatorname{Gal}(k / K)$, and so $\phi([0])$ descends to $K ; A(K)$ is nonempty. By uniqueness of group structures on abelian varieties, we see that the group law on $A$ also descends to $\underline{A} ; A$ descends to $K$ as an abelian variety.

Definition 4.2. Let $k$ be an algebraically closed field, and $K \subseteq k$ a perfect sub-field such that $k / K$ is algebraic. Let $X$ be a smooth projective variety over $k$, equipped with $k / K$ descent data. A Galois equivariant algebraic representative for $\mathrm{A}^{i}(X)$ is a pair $\left(\mathrm{Ab}_{\mathrm{Gal}(k / K)}^{i}(X), \phi_{X, \operatorname{Gal}(k / K)}\right)$ which is initial among all pairs $(A, \phi)$ consisting of an abelian variety $A / k$ with $k / K$ descent datum and a Galois-equivariant regular homomorphism $\phi: \mathrm{A}^{i}(X) \rightarrow A(k)$.

Remark 4.3. Note that for a pointed smooth integral $k$-variety $T$, and a codimension $i$ cycle $Z$ on $T \times_{k} X$, the following diagram is commutative for a Galois equivariant regular homomorphism $\phi: \mathrm{A}^{i}(X) \rightarrow A(k):$

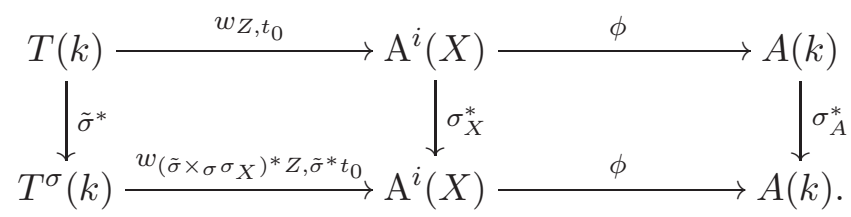


Moreover, for a smooth integral $k$-variety $T$ with descent datum, together with a $\operatorname{Gal}(k / K)$-invariant marked point $t_{0}$, and a codimension $i$ cycle $Z$ on $T \times_{k} X$ such that the descent data for $T$ and $X$ induces descent data for $Z$, the following diagram is commutative for a Galois invariant regular homomorphism $\phi: \mathrm{A}^{i}(X) \rightarrow A(k)$ :

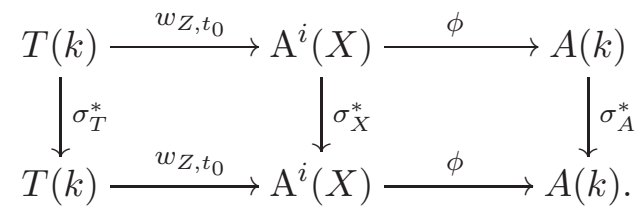

This says that the morphism of $k$-varieties $T \rightarrow A$ inducing $\phi \circ w_{Z}$ descends to $K$.

We now prove some basic results about Galois equivariant regular morphisms. The main result we want is that for smooth projective varieties over a perfect field $K$, algebraic representatives of the variety over the algebraic closure give Galois equivariant algebraic representatives ; in particular, they descend to $K$.

Theorem 4.4. Let $X / k$ be a smooth projective variety over an algebraically closed field $k$, and suppose that $\mathrm{A}^{i}(X)$ has an algebraic representative $\left(\mathrm{Ab}^{i}(X), \phi_{X}^{i}\right)$. Let $K \subseteq k$ be a perfect field such that $k / K$ is algebraic. Then each $k / K$ descent datum on $X$ induces a $k / K$ descent datum on $\mathrm{Ab}^{i}(X)$. Moreover, with respect to these descent data, $\phi_{X}^{i}$ is $\operatorname{Gal}(k / K)$-equivariant, and the pair $\left(\mathrm{Ab}^{i}(X), \phi_{X}^{i}\right)$ is a Galois equivariant algebraic representative.

Remark 4.5. Note that by Remark 4.1, $\mathrm{Ab}^{i}(X)$ descends to an abelian variety $\underline{\mathrm{Ab}}^{i}(X)$ over $K$.

The proof uses the universal property of $\mathrm{Ab}^{i}(X)$ to transfer descent data for $X$ to descent data for $\mathrm{Ab}^{i}(X)$. Before giving the proof, we introduce one more notion that we will utilize.

Definition 4.6. Let $X$ be a smooth projective variety over an algebraically closed field $k$, and let $\sigma \in \operatorname{Aut}(k)$. Given an abelian variety $A$ over $k$, a homomorphism of groups

$$
\mathrm{A}^{i}(X) \stackrel{\phi}{\longrightarrow} A(k)
$$

is said to be $\sigma$-regular if for every pair $(T, Z)$ with $T$ a pointed smooth integral $k$-variety, and $Z \subseteq T \times_{k} X$ a relative codimension $i$ cycle over $T$, the composition

$$
T(k) \stackrel{w_{Z}}{\longrightarrow} \mathrm{A}^{i}(X) \stackrel{\phi}{\longrightarrow} A(k)
$$

is induced by a morphism $g: T \rightarrow A$ over $\sigma$; by this (i.e., $\phi \circ w_{Z}$ being induced by the morphism $g$ over $\sigma$ ) we mean there is a commutative diagram of morphisms of schemes

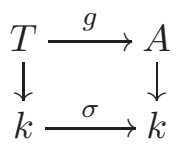

such that for each $k$-point $t: \operatorname{Spec} k \rightarrow T$, we have $\left(\phi \circ w_{Z}\right)(t)=g_{*}(t):=g \circ t \circ \sigma^{-1}$.

Lemma 4.7. Let $X$ be a smooth projective variety over an algebraically closed field $k$, and let $\sigma \in \operatorname{Aut}(k)$. Suppose that $\left(\mathrm{Ab}^{i}(X), \phi_{X}^{i}\right)$ is an algebraic representative for $\mathrm{A}^{i}(X)$. Given an abelian variety $A / k$ and a $\sigma$-regular homomorphism $\phi: \mathrm{A}^{i}(X) \rightarrow A(k)$, there exists a unique morphism $f: \operatorname{Ab}^{i}(X) \rightarrow A$ over $\sigma$ such that $\phi=f_{*} \circ \phi_{X}^{i}$.

Proof. Let $\phi: \mathrm{A}^{i}(X) \rightarrow A(k)$ be a $\sigma$-regular homomorphism. Consider the morphisms

$$
A(k) \stackrel{\tilde{\sigma}^{*}}{\longrightarrow} A^{\sigma}(k), \quad A \stackrel{\tilde{\sigma}^{-1}}{\longrightarrow} A^{\sigma},
$$


with the latter over $\sigma^{-1} \in \operatorname{Aut}(k)$, and where given a $k$-point $a: \operatorname{Spec} k \rightarrow A$, we define $\tilde{\sigma}^{*}(a):=$ $\left(\tilde{\sigma}^{-1}\right)_{*}(a)=\tilde{\sigma}^{-1} a \sigma$.

The composition $\tilde{\sigma}^{*} \circ \phi: \mathrm{A}^{i}(X) \rightarrow A^{\sigma}(k)$ is a regular homomorphism. Indeed, given a triple $\left(T, Z, t_{0}\right)$ with $T$ a smooth integral $k$-variety, $Z$ a codimension $i$ cycle on $T \times_{k} X$, and $t_{0}:$ Spec $k \rightarrow T$ a $k$-point, there is by definition a morphism $g: T \rightarrow A$ over $\sigma$ such that for each $k$-point $t:$ Spec $k \rightarrow$ $T$ we have $\left(\phi w_{Z}\right)(t)=g t \sigma^{-1}$. Therefore, $\left(\tilde{\sigma}^{*} \phi w_{Z}\right)(t)=\tilde{\sigma}^{*}\left(g t \sigma^{-1}\right)=\tilde{\sigma}^{-1}\left(g t \sigma^{-1}\right) \sigma=\tilde{\sigma}^{-1} g t$, so that $\tilde{\sigma}^{*} \phi w_{Z}$ is induced by $\tilde{\sigma}^{-1} g: T \rightarrow A^{\sigma}$, and we have established that $\tilde{\sigma}^{*} \phi$ is a regular homomorphism.

It follows from the universal property of the algebraic representative that there is a unique morphism $\tilde{f}: \mathrm{Ab}^{i}(X) \rightarrow A^{\sigma}$ of abelian varieties over $k$, such that $\tilde{\sigma}^{*} \circ \phi=\tilde{f} \circ \phi_{X}^{i}$ on $k$-points. The morphism $f:=\tilde{\sigma} \circ \tilde{f}$ over $\sigma$ satisfies $\phi=\tilde{\sigma}_{*} \tilde{\sigma}^{*} \phi=\tilde{\sigma}_{*} \tilde{f} \phi_{X}^{i}=f_{*} \phi_{X}^{i}$. Here we are using that $\tilde{\sigma}_{*} \tilde{f}=f_{*}$ on $k$-points; indeed, given a $k$-point $a: \operatorname{Spec} k \rightarrow \operatorname{Ab}^{i}(X)$, we have $\tilde{\sigma}_{*} \tilde{f}(a)=\tilde{\sigma}_{*}(\tilde{f} a)=$ $\tilde{\sigma} \tilde{f} a \sigma^{-1}=f a \sigma^{-1}=f_{*}(a)$. The uniqueness of $f$ follows again from the universal property of the algebraic representative.

Proof of Theorem 4.4. We start by showing that $k / K$ descent data for $X$ induces $k / K$ descent data for $\mathrm{Ab}^{i}(X)$. The first claim is that the composition $\phi_{X}^{i} \circ \sigma_{X}^{*}: \mathrm{A}^{i}(X) \rightarrow \mathrm{Ab}^{i}(X)(k)$ is $\sigma^{-1}$-regular. Indeed, suppose we are given $T / k$, a smooth integral pointed variety, and $Z$ a codimension $i$ cycle on $T \times_{k} X$. It was shown in (4.2) that we have a commutative diagram

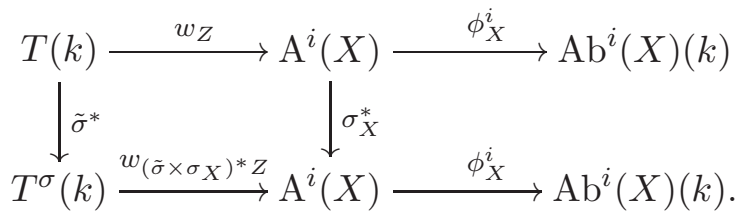

Let $g^{\prime}: T^{\sigma} \rightarrow \mathrm{Ab}^{i}(X)$ be the morphism of $k$-varieties inducing the bottom row of the diagram, and set $g:=g^{\prime} \circ \tilde{\sigma}^{-1}: T \rightarrow \mathrm{Ab}^{i}(X)$ to be the induced morphism over $\sigma^{-1}$. For a $k$-point $t:$ Spec $k \rightarrow T$ we have

$$
\left(\phi_{X}^{i} \sigma_{X}^{*} w_{Z}\right)(t)=\left(\phi_{X}^{i} w_{\left(\tilde{\sigma} \times \sigma_{X}\right)^{*} Z^{*}} \tilde{\sigma}^{*}(t)=g^{\prime}\left(\tilde{\sigma}^{*} t\right)=g^{\prime} \tilde{\sigma}^{-1} t \sigma=g t \sigma=g_{*}(t) .\right.
$$

Therefore we have established that $\phi_{X}^{i} \sigma_{X}^{*}$ is $\sigma^{-1}$-regular.

By virtue of the previous lemma, there is a unique morphism $\left(\sigma_{\mathrm{Ab}}\right)^{-1}: \mathrm{Ab}^{i}(X) \rightarrow \mathrm{Ab}^{i}(X)$ over $\sigma^{-1}$ making the following diagram commute :

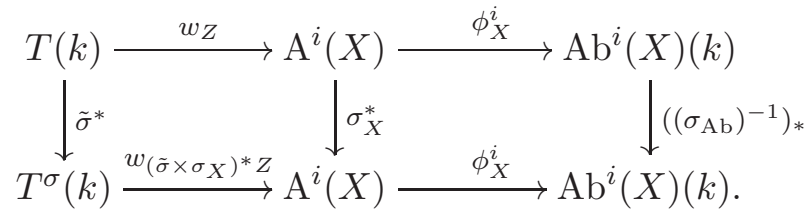

Using the universal property of the algebraic representative, one can see that the morphisms $\left(\sigma_{\mathrm{Ab}}\right)^{-1}: \mathrm{Ab}^{i}(X) \rightarrow \mathrm{Ab}^{i}(X)$ are isomorphisms, and that the morphisms $\sigma_{\mathrm{Ab}}:=\left(\left(\sigma_{\mathrm{Ab}}\right)^{-1}\right)^{-1}$ define a lift of the action of $\operatorname{Gal}(k / K)$ to $\mathrm{Ab}^{i}(X)$. In particular, the $\sigma_{\mathrm{Ab}}$ provide a $k / K$ descent datum for $\mathrm{Ab}^{i}(X)$. Finally, the commutativity of the diagram

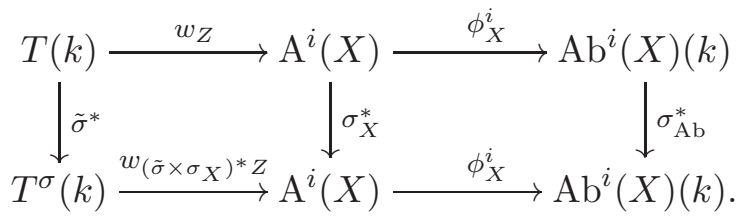

shows that $\phi_{X}^{i}$ is $\operatorname{Gal}(k / K)$-equivariant, and therefore that $\left(\operatorname{Ab}^{i}(X), \phi_{X}^{i}\right)$ is a Galois equivariant algebraic representative. 
4.4. Some structure results for algebraic representatives. We would also like to extend some basic structure results from Murre [Mur85] to the case of Galois equivariant regular homomorphisms ; a crucial ingredient is the following technical, yet general, proposition extending a well known result of Weil Wei54, Lem. 9] over an algebraically closed field to the setting of arbitrary perfect fields :

Proposition 4.8 ([ACMVc] $)$. Let $\underline{X} / K$ be a scheme of finite type over a perfect field $K$ and let $X$ be the base-change of $\underline{X}$ to an algebraic closure $k$ of $K$. If $\alpha \in \mathrm{CH}^{i}(X)$ is algebraically trivial, then there exist an abelian variety $\underline{A} / K$, a cycle $\underline{Z}$ on $\underline{A} \times_{K} \underline{X}$, and a pair of $k$-points $t_{1}$, $t_{0}$ on $\underline{A}$ such that $\alpha=Z_{t_{1}}-Z_{t_{0}}$.

Lemma 4.9 (Mur85, Lem. 1.6.2, Cor. 1.6.3]). Let $k$ be an algebraically closed field, let $K \subseteq k$ be a perfect sub-field such that $k / K$ is algebraic, let $X$ be a smooth projective variety over $k$, let $A$ be an abelian variety over $k$, and assume that each is equipped with $k / K$-descent data. Now let $\phi: \mathrm{A}^{i}(X) \rightarrow A(k)$ be a $\mathrm{Gal}(k / K)$-equivariant regular homomorphism. Then:

(a) There is an abelian subvariety $\underline{A}^{\prime} \subseteq \underline{A}$ defined over $K$ such that $\operatorname{Im}(\phi)=A^{\prime}(k)$.

(b) There is an abelian variety $\underline{D}$ defined over $K$ and a cycle $\underline{Z} \in \mathrm{CH}^{i}\left(\underline{D} \times_{K} \underline{X}\right)$ such that the composition $D(k) \stackrel{w_{Z}}{\longrightarrow} \mathrm{A}^{i}(X) \stackrel{\phi}{\longrightarrow} A(k)$ surjects onto $\operatorname{Im}(\phi)$ and is induced by a $K$-morphism of abelian varieties $\underline{D} \rightarrow \underline{A}$.

(c) If $\phi$ is surjective, then there is an abelian variety $\underline{B}$ defined over $K$ and a cycle $\underline{Z} \in \mathrm{CH}^{i}(\underline{B} \times K$ $\underline{X})$ such that the composition $B(k) \stackrel{w_{Z}}{\longrightarrow} \mathrm{A}^{i}(X) \stackrel{\phi}{\longrightarrow} A(k)$ is induced by a K-isogeny of abelian varieties $\underline{B} \rightarrow \underline{A}$.

(d) If $\phi$ is surjective, then there exists a cycle $\underline{Z} \in \mathrm{CH}^{i}(\underline{A} \times K \underline{X})$ such that the composition

$$
A(k) \stackrel{w_{Z}}{\longrightarrow} \mathrm{A}^{i}(X) \stackrel{\phi}{\longrightarrow} A(k)
$$

is induced by the $K$-morphism $r \cdot \operatorname{Id}_{\underline{A}}: \underline{A} \rightarrow \underline{A}$, for some non-zero integer $r$.

Proof. This is Mur85, Lem. 1.6.2, Cor. 1.6.3] in the case $K=k$. We will see that, with the arithmetic input of Proposition 4.8, the same proof establishes the present lemma. As (a) follows from the proof of (b), we begin by proving (b).

(b) The key point is that given $\alpha \in \operatorname{Im}(\phi)$, there exist an abelian variety $\underline{D}$ defined over $K$ and a $\underline{Y} \in \mathrm{CH}^{i}(\underline{D} \times K \underline{X})$ such that $\alpha \in \operatorname{Im}\left(\phi \circ w_{Y}\right)$. In the case $k=K$, this is [Wei54, Lem. 9]; in the case $k \neq K$, this is Proposition 4.8.

Now for all such pairs $(\underline{D}, \underline{Y})$ consider the abelian sub-varieties $\operatorname{Im}\left(\phi \circ w_{Y}\right)$. Note that, since we are assuming that $\phi$ is $\operatorname{Gal}(k / K)$-equivariant, Remark 4.3 says that $\phi \circ w_{Y}$ descends to $K$. Clearly we can take a pair $\left(\underline{D}^{\prime}, \underline{Y}^{\prime}\right)$ such that $\operatorname{Im}\left(\phi \circ w_{Y^{\prime}}\right)$ is of maximal dimension. The claim is that $\operatorname{Im}\left(\phi \circ w_{Y^{\prime}}\right)=\operatorname{Im}(\phi)$. If not, let $\phi(\alpha) \notin \operatorname{Im}\left(\phi \circ w_{Y^{\prime}}\right)$, then by the assertion of the first paragraph, there exists a pair $\left(\underline{D}^{\prime \prime}, \underline{Y}^{\prime \prime}\right)$ such that $\phi(\alpha) \in \operatorname{Im}\left(\phi \circ w_{Y^{\prime \prime}}\right)$. Now consider $\underline{D}^{\prime \prime \prime}:=\underline{D}^{\prime} \times_{K} \underline{D}^{\prime \prime}$ and the projections $\pi^{\prime}: \underline{D}^{\prime} \times_{K} \underline{D}^{\prime \prime} \times_{K} \underline{X} \rightarrow \underline{D}^{\prime} \times_{K} \underline{X}$ and $\pi^{\prime \prime}: \underline{D}^{\prime} \times_{K} \underline{D}^{\prime \prime} \times_{K} \underline{X} \rightarrow \underline{D}^{\prime \prime} \times_{K} \underline{X}$. Take

$$
\underline{Y}^{\prime \prime \prime}:=\pi^{\prime *} \underline{Y}^{\prime}+\pi^{\prime \prime *} \underline{Y}^{\prime \prime} \text {. }
$$

Then $\operatorname{Im}\left(\phi \circ w_{Y^{\prime \prime \prime}}\right) \supset \operatorname{Im}\left(\phi \circ w_{Y^{\prime}}\right)$ and $\operatorname{Im}\left(\phi \circ w_{Y^{\prime \prime \prime}}\right) \supset \operatorname{Im}\left(\phi \circ w_{Y^{\prime \prime}}\right)$, a contradiction, proving the claim, and thus (b).

(c) We have a surjection $\underline{D} \rightarrow \underline{A}$, and there exists a $K$-sub-abelian variety $\underline{B} \subseteq \underline{D}$ such that the induced map $\underline{B} \rightarrow \underline{A}$ is an isogeny. The restricted cycle $\underline{Z} \mid \underline{B} \times_{K} \underline{X}$ provides the desired cycle.

(d) This follows from (c). The dual isogeny $g: \underline{A} \rightarrow \underline{B}$ has the property that there is a non-zero integer $r \in \mathbb{Z}$ such that the composition $\underline{A} \rightarrow \underline{B} \rightarrow \underline{A}$ is given by $r \cdot \operatorname{Id}_{\underline{A}}$. The cycle $\left(g \times_{K} \operatorname{Id}_{\underline{X}}\right)^{*} \underline{Z}$ gives the desired cycle. 
In light of Murre's results [Mur85] (see Theorem 3.4), we have the following consequence (see also Corollary 5.2 and Remark 5.4, which establishes a stronger statement for cohomology with $\mathbb{Q}_{\ell}$-coefficients, under the hypothesis $K \subseteq \mathbb{C}$ ).

Corollary 4.10. Let $X$ be a smooth projective variety over a perfect field $K$. Let $\underline{\mathrm{Ab}}^{2}(X) / K$ be the model of $\mathrm{Ab}^{2}(X)$ from Theorem 4.4. There is a natural inclusion of $\mathrm{Gal}(k / K)$-representations

$$
H^{1}\left(\underline{\mathrm{Ab}}^{2}(X){ }_{k}, \mathbb{Q}_{\ell}\right) \hookrightarrow H^{3}\left(X, \mathbb{Q}_{\ell}(1)\right) .
$$

For almost all $\ell$ there is an inclusion $\left.H^{1}\left({\underline{\underline{\mathrm{b}^{2}}}}^{(X}\right)_{k}, \mathbb{Z}_{\ell}\right) \hookrightarrow H^{3}\left(X, \mathbb{Z}_{\ell}(1)\right)$ of $\operatorname{Gal}(k / K)$-modules.

Proof. We aim to show that the two inclusions (3.4), i.e., $T_{\ell} \mathrm{Ab}^{2}(X) \hookrightarrow T_{\ell} \mathrm{A}^{2}(X)$, and (3.5), i.e., $T_{\ell} \mathrm{CH}^{2}(X) \hookrightarrow H^{3}\left(X, \mathbb{Z}_{\ell}(2)\right)$ constructed by Murre are $\operatorname{Gal}(k / K)$-equivariant. The second inclusion (3.5) can be seen to be equivariant since the map is constructed via natural maps of sheaves, all of which have natural $\mathrm{Gal}(k / K)$-actions. Let us now show that the first inclusion (3.4) can be taken to be equivariant. Using the modification of [Mur85, Cor. 1.6.3] given in Lemma 4.9, there is a cycle $\underline{Z} \in \mathrm{CH}^{2}\left(\underline{\mathrm{Ab}}^{2}(X) \times_{K} \underline{X}\right)$ such that

$$
\phi_{X}^{2} \circ w_{Z}: \operatorname{Ab}^{2}(X)(k) \rightarrow \mathrm{A}^{2}(X) \rightarrow \operatorname{Ab}^{2}(X)(k)
$$

is induced by a $K$-isogeny $f: \underline{\mathrm{Ab}}^{2}(X) \rightarrow \underline{\mathrm{Ab}}^{2}(X)$. We also saw in the proof of Theorem 4.4 that both $w_{Z}$ and $\phi_{X}^{2}$ are Galois equivariant. Now take a prime number $\ell$ such that $(\ell, \operatorname{deg}(f))=1$ and $\ell$ is invertible in $k$; on points of order $\ell^{\nu}$ we have

$$
f: \mathrm{Ab}^{2}(X)\left[\ell^{\nu}\right] \stackrel{\sim}{\rightarrow} \mathrm{Ab}^{2}(X)\left[\ell^{\nu}\right],
$$

as Galois representations, for all $\nu>0$. Therefore $\mathrm{Ab}^{2}(X)\left[\ell^{\nu}\right]$ is a direct summand of the Galois representation $\mathrm{A}^{2}(X)\left[\ell^{\nu}\right]$ and we obtain a Galois equivariant inclusion

$$
\operatorname{Ab}^{2}(X)\left[\ell^{\nu}\right] \hookrightarrow \mathrm{A}^{2}(X)\left[\ell^{\nu}\right] .
$$

Taking the associated Tate modules completes the proof.

\section{Phantoms via algebraic Representatives}

We now prove Theorem $\mathrm{B}$ and complete the proof of Theorem $\mathrm{A}$, by showing in Theorem 5.1 that the image of the Abel-Jacobi map in the intermediate Jacobian $J^{3}\left(X_{\mathbb{C}}\right)$ descends to a field of definition of $X$.

Theorem 5.1. Suppose $X$ is a smooth projective variety over a field $K \subseteq \mathbb{C}$, and $n$ is a non-negative integer. Assume that $\mathrm{A}^{n+1}\left(X_{\mathbb{C}}\right)$ admits $\left(J_{a}^{2 n+1}\left(X_{\mathbb{C}}\right), A J\right)$ as an algebraic representative, where $J_{a}^{2 n+1}\left(X_{\mathbb{C}}\right)$ is the image of the Abel-Jacobi map AJ: $\mathrm{A}^{n+1}\left(X_{\mathbb{C}}\right) \rightarrow J^{2 n+1}\left(X_{\mathbb{C}}\right)$. Then $J_{a}^{2 n+1}\left(X_{\mathbb{C}}\right)$ has a distinguished model $J$ over $K$ making $A J: \mathrm{A}^{n+1}\left(X_{\mathbb{C}}\right) \rightarrow J_{a}^{2 n+1}\left(X_{\mathbb{C}}\right)$ an $\operatorname{Aut}(\mathbb{C} / K)$-equivariant homomorphism, and there is a correspondence $\gamma$ on $\widehat{J} \times_{K} X$ inducing for each prime number $\ell$ an inclusion of $\mathrm{Gal}(K)$-representations

$$
H^{1}\left(\widehat{J}_{\bar{K}}, \mathbb{Q}_{\ell}\right) \stackrel{\gamma_{*}}{\hookrightarrow} H^{2 n+1}\left(X_{\bar{K}}, \mathbb{Q}_{\ell}(n)\right),
$$

with image $\mathrm{N}^{n} H^{2 n+1}\left(X_{\bar{K}}, \mathbb{Q}_{\ell}(n)\right)$. Consequently, if in addition $H^{2 n+1}\left(X_{\mathbb{C}}, \mathbb{Q}\right)$ is of geometric coniveau $n$, then (5.1) is an isomorphism.

Proof. Let $X$ be a smooth projective variety defined over $K \subseteq \mathbb{C}$ and assume that $\mathrm{A}^{n+1}\left(X_{\mathbb{C}}\right)$ admits an algebraic representative $\left(\mathrm{Ab}^{n+1}\left(X_{\mathbb{C}}\right), \phi_{X_{\mathbb{C}}}^{n+1}\right)$. We have seen in Theorem 3.7 that $\mathrm{A}^{n+1}\left(X_{\bar{K}}\right)$ admits an algebraic representative $\left(\mathrm{Ab}^{n+1}\left(X_{\bar{K}}\right), \phi_{X_{\bar{K}}}^{n+1}\right)$ with $\mathrm{Ab}^{n+1}\left(X_{\bar{K}}\right)_{\mathbb{C}} \cong \mathrm{Ab}^{n+1}\left(X_{\mathbb{C}}\right)$, and in 
Theorem 4.4 that $\mathrm{Ab}^{n+1}\left(X_{\bar{K}}\right)$ admits a distinguished model $J$ over $K$. (Note that the distinguished model $J$ over $K$ is indeed an abelian variety; see Remark 4.1.) We also saw in Theorem 3.7 that $\left(\mathrm{Ab}^{n+1}\left(X_{\bar{K}}\right), \phi_{X_{\bar{K}}}^{n+1}\right)$ is the $\mathbb{C} / \bar{K}$-trace of $\left(\mathrm{Ab}^{n+1}\left(X_{\mathbb{C}}\right), \phi_{X_{\mathbb{C}}}^{n+1}\right)$ and in Theorem 4.4 that $\mathrm{Ab}^{n+1}\left(X_{\bar{K}}\right)$ is equipped with Galois descent datum making $\phi_{X_{\bar{K}}}^{n+1}$ Galois-equivariant. Since the $\mathbb{C} / \bar{K}$-trace is constructed by fpqc descent Con06, we see that $\phi_{X_{\mathbb{C}}^{n+1}}^{n}: \mathrm{A}^{n+1}\left(X_{\mathbb{C}}\right) \rightarrow \mathrm{Ab}^{n+1}\left(X_{\mathbb{C}}\right)$ is $\operatorname{Aut}(\mathbb{C} / K)$-equivariant. Moreover, since the regular homomorphism $\phi_{X_{\mathbb{C}}}^{n+1}$ is surjective, the model $J$ is distinguished in the sense that it is uniquely determined by the natural Galois action on $\mathrm{A}^{n+1}\left(X_{\mathbb{C}}\right)$.

We now make a general observation. Let $X_{\mathbb{C}}$ be a smooth projective variety defined over $\mathbb{C}$. Given an abelian variety $A_{\mathbb{C}}$ over $\mathbb{C}$ and a correspondence $Z_{\mathbb{C}} \in \mathrm{CH}^{n+1}\left(A_{\mathbb{C}} \times_{\mathbb{C}} X_{\mathbb{C}}\right)$, then using the functoriality of the Abel-Jacobi map with respect to the action of correspondences we have a commutative diagram

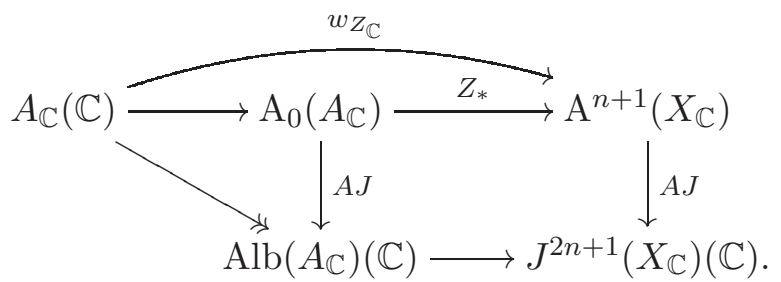

The differential of the map $\operatorname{Alb}\left(A_{\mathbb{C}}\right) \rightarrow J^{2 n+1}\left(X_{\mathbb{C}}\right)$ in the bottom row is the complexification of the map

$$
Z_{*}: H^{2 \operatorname{dim} A-1}\left(A_{\mathbb{C}}, \mathbb{Q}\left(\operatorname{dim} A_{\mathbb{C}}-1\right)\right) \rightarrow H^{2 n+1}\left(X_{\mathbb{C}}, \mathbb{Q}(n)\right),
$$

induced by the correspondence $Z_{\mathbb{C}}$ (e.g., Voi07, Thm. 12.17]).

The natural identification $H^{1}\left(\widehat{A}_{\mathbb{C}}, \mathbb{Q}\right) \rightarrow H^{2 \operatorname{dim} A-1}\left(A_{\mathbb{C}}, \mathbb{Q}\left(\operatorname{dim} A_{\mathbb{C}}-1\right)\right)$ can be obtained via correspondences as the composition of isomorphisms $\Theta_{*} \circ \Gamma_{\lambda *}$, where $\Gamma_{\lambda}$ is the graph of a polarization $\lambda: \widehat{A}_{\mathbb{C}} \rightarrow A_{\mathbb{C}}$, and $\Theta_{*}: H^{1}\left(A_{\mathbb{C}}, \mathbb{Q}\right) \rightarrow H^{2 \operatorname{dim} A-1}\left(A_{\mathbb{C}}, \mathbb{Q}\left(\operatorname{dim} A_{\mathbb{C}}-1\right)\right)$ is induced by the $\left(\operatorname{dim} A_{\mathbb{C}}-1\right)$ fold intersection $\Theta$ of the class of the dual polarization on $A_{\mathbb{C}}$. In conclusion, we have a morphism

$$
H^{1}\left(\widehat{A}_{\mathbb{C}}, \mathbb{Q}\right) \stackrel{Z_{*} \circ \Theta_{*} \circ \Gamma_{\lambda *}}{\longrightarrow} H^{2 n+1}\left(X_{\mathbb{C}}, \mathbb{Q}(n)\right) .
$$

Finally consider the special case where $\left(A_{\mathbb{C}}, \phi\right)=\left(J_{a}^{2 n+1}, A J\right)$, and where the cycle $Z_{\mathbb{C}}$ is taken as in [Mur85, Cor. 1.6.3] (see Lemma 4.9)(d)) so that $A J \circ w_{Z_{\mathbb{C}}}$ is given by $r \cdot \operatorname{Id}_{J_{a}^{2 n+1}}$ for some non-zero integer $r$. The diagram (5.2) then shows that (5.4) is injective, with image $\mathrm{N}^{n} H^{2 n+1}\left(X_{\mathbb{C}}, \mathbb{Q}\right)$.

With this set-up, Lemma 4.9 implies that there exists a cycle $Z \in \mathrm{CH}^{n+1}\left(J \times_{K} X\right)$ such that the composition

$$
\operatorname{Ab}^{n+1}\left(X_{\bar{K}}\right) \stackrel{w_{Z_{\bar{K}}}}{\longrightarrow} \mathrm{A}^{n+1}\left(X_{\bar{K}}\right) \stackrel{\phi_{X_{\bar{K}}}^{n+1}}{\longrightarrow} \operatorname{Ab}^{n+1}\left(X_{\bar{K}}\right)
$$

is induced by the $K$-morphism $r \cdot \operatorname{Id}_{J}: J \rightarrow J$ for some non-zero integer $r$, and the maps $w_{Z_{\bar{K}}}$ and $\phi_{X_{K}}^{n+1}$ are $\operatorname{Gal}(K)$-equivariant. Let $\lambda: \widehat{J} \rightarrow J$ be a polarization and let $\Theta$ be the $(\operatorname{dim} J-1)$-fold self-intersection of the class of the polarization on $J$. The correspondence $Z \circ \Theta \circ \Gamma_{\lambda}$, defined over $K$, induces a morphism of $\mathrm{Gal}(K)$-representations

$$
H^{1}\left(\widehat{J}_{\bar{K}}, \mathbb{Q}_{\ell}\right) \stackrel{Z_{*} \circ \Theta_{*} \circ \Gamma_{\lambda *}}{\longrightarrow} H^{2 n+1}\left(X_{\bar{K}}, \mathbb{Q}_{\ell}(n)\right) .
$$

Finally, if we assume further that $\left(J_{a}^{2 n+1}\left(X_{\mathbb{C}}\right), A J\right) \cong\left(\mathrm{Ab}^{n+1}\left(X_{\mathbb{C}}\right), \phi_{X_{\mathbb{C}}}^{n+1}\right)$, then (5.4) and the comparison isomorphisms (1.3) imply that the map (5.5) is an inclusion, with image $\mathrm{N}^{n} H^{2 n+1}\left(X_{\bar{K}}, \mathbb{Q}_{\ell}(n)\right)$. The comparison isomorphisms (1.3) also show that $H^{2 n+1}\left(X_{\mathbb{C}}, \mathbb{Q}\right)$ is of 
geometric coniveau $n$ if and only if $H^{2 n+1}\left(X_{\bar{K}}, \mathbb{Q}_{\ell}\right)$ is of geometric coniveau $n$; therefore (5.5) is an isomorphism under the hypotheses given in the theorem.

Corollary 5.2. Suppose that $X$ is a smooth projective variety over a field $K \subseteq \mathbb{C}$. The abelian variety $J_{a}^{3}\left(X_{\mathbb{C}}\right)$ has a distinguished model $J$ over $K$. There is a correspondence $\gamma$ on $\widehat{J} \times_{K} X$ such that for each prime number $\ell$ the correspondence induces an inclusion of $\mathrm{Gal}(K)$-representations

$$
H^{1}\left(\widehat{J}_{\bar{K}}, \mathbb{Q}_{\ell}\right) \stackrel{\gamma_{*}}{\hookrightarrow} H^{3}\left(X_{\bar{K}}, \mathbb{Q}_{\ell}(1)\right)
$$

with image $\mathrm{N}^{1} H^{3}\left(X_{\bar{K}}, \mathbb{Q}_{\ell}(1)\right)$. Consequently, if $H^{3}\left(X_{\mathbb{C}}, \mathbb{Q}\right)$ is of geometric coniveau 1 (e.g., if $X_{\mathbb{C}}$ is a uni-ruled threefold), then (5.6) is an isomorphism.

Proof. This follows from Theorem 5.1] and [Mur85] (see Theorem 3.4). That a uni-ruled threefold $X_{\mathbb{C}}$ has $H^{3}\left(X_{\mathbb{C}}, \mathbb{Q}\right)$ of geometric coniveau 1 follows, via a decomposition of the diagonal argument BS83, from the fact that $\mathrm{CH}_{0}\left(X_{\mathbb{C}}\right)$ is supported on a surface.

Remark 5.3. Unlike the approach taken to prove Theorem 2.1, the approach to proving Theorem5.1 using Murre's results [Mur85] does not seem to provide a splitting for the inclusion (5.6). However, since we are taking $2 n+1=3$, the arguments in the proof of Theorem 2.1 do provide a splitting for (5.6), induced by an algebraic correspondence if $K$ is finitely generated. Indeed, let $J^{\prime}$ be the abelian variety over $K$ and $\gamma^{\prime}$ be the correspondence provided by Theorem 2.1. The inclusion (5.6) and the inclusion $\gamma_{*}^{\prime}: H^{1}\left(J_{\bar{K}}^{\prime}, \mathbb{Q}_{\ell}\right) \hookrightarrow H^{3}\left(X_{\bar{K}}, \mathbb{Q}_{\ell}(1)\right)$ have the same image, namely $\mathrm{N}^{1} H^{3}\left(X_{\bar{K}}, \mathbb{Q}_{\ell}(1)\right)$. Therefore $H^{1}\left(J_{\bar{K}}, \mathbb{Q}_{\ell}\right)$ and $H^{1}\left(J_{\bar{K}}^{\prime}, \mathbb{Q}_{\ell}\right)$ are isomorphic as $\mathrm{Gal}(K)$-representations. The splitting of $\gamma_{*}^{\prime}$ from Theorem 2.1 then provides a splitting of (5.6). If moreover $K$ is finitely generated, then $J$ and $J^{\prime}$ are $K$-isogenous, in which case composing the the graph of such an isogeny with the correspondence from Theorem 2.1 that gives the splitting of $\gamma_{*}^{\prime}$ yields a correspondence splitting (5.6).

Remark 5.4. Comparing Corollary 5.2 to Corollary 4.10, we see that by assuming that $K \subseteq \mathbb{C}$ and by using the functoriality of the Abel-Jacobi map with respect to the action of correspondences, we are able to obtain the inclusion of $\mathrm{Gal}(K)$-representations via a correspondence, and to characterize its image in terms of the geometric coniveau filtration.

\section{Complements on phantoms and algebraic Representatives}

In this section we discuss specialization of phantom abelian varieties and algebraic representatives. This has particular relevance to the question of phantoms from the perspective of Honda-Tate theory. Motivated by Theorem [5.1, we also establish some results on algebraic representatives for higher codimension cycles.

\subsection{Phantoms, algebraic representatives, and specializations.}

Lemma 6.1. Let $S=\operatorname{Spec} R$ be a discrete valuation ring with generic point $\eta=\operatorname{Spec} K$ and special point $0=\operatorname{Spec} \kappa$, and let $\ell$ be a prime number invertible in $\kappa$. Let $X / S$ be a smooth, projective scheme, and let $A / \eta$ be an abelian variety equipped with a $\mathrm{Gal}(K)$-equivariant inclusion

$$
H^{1}\left(A_{\bar{\eta}}, \mathbb{Q}_{\ell}\right) \longleftrightarrow H^{2 n+1}\left(X_{\bar{\eta}}, \mathbb{Q}_{\ell}(n)\right) .
$$

(a) Then $A$ extends to an abelian scheme $A / S$.

(b) Suppose the inclusion (6.1) has image $\mathrm{N}^{n} H^{2 n+1}\left(X_{\bar{\eta}}, \mathbb{Q}_{\ell}\right)$ and that $\operatorname{dim} \mathrm{N}^{n} H^{2 n+1}\left(X_{\overline{0}}, \mathbb{Q}_{\ell}\right)=$ $\operatorname{dim} \mathrm{N}^{n} H^{2 n+1}\left(X_{\bar{\eta}}, \mathbb{Q}_{\ell}\right)$. Then specialization induces an inclusion

$$
H^{1}\left(A_{\overline{0}}, \mathbb{Q}_{\ell}\right) \longleftrightarrow H^{2 n+1}\left(X_{\overline{0}}, \mathbb{Q}_{\ell}(n)\right),
$$

with image $\mathrm{N}^{n} H^{2 n+1}\left(X_{\overline{0}}, \mathbb{Q}_{\ell}\right)$. Moreover, if (6.1) is realized by a correspondence on $A \times_{\eta} X_{\eta}$, then (6.2) is realized by the specialization of the correspondence on $A_{0} \times_{0} X_{0}$. 
(c) If $A_{\eta}$ is a phantom for $X_{\eta}$ (i.e., (6.1) is an isomorphism), then $A_{0}$ is a phantom for $X_{0}$ (i.e., (6.2) is an isomorphism).

Proof. (a) Since $X_{\eta}$ has good reduction, it follows that $H^{2 n+1}\left(X_{\bar{\eta}}, \mathbb{Q}_{\ell}\right)$ is unramified as a representation of $\operatorname{Gal}(K)$, and thus so is $H^{1}\left(A_{\bar{\eta}}, \mathbb{Q}_{\ell}\right)$. The Néron-Ogg-Shafarevich criterion shows $A$ extends to $S$.

(b) This follows from the canonical isomorphism $H^{r}\left(Y_{\bar{\eta}}, \mathbb{Q}_{\ell}\right) \cong H^{r}\left(Y_{\overline{0}}, \mathbb{Q}_{\ell}\right)$ provided by proper base change for any smooth projective $Y / S$. More precisely, since specialization is compatible with intersection [Ful98, 20.3.5], the specialization isomorphism gives an inclusion $\mathrm{N}^{n} H^{2 n+1}\left(X_{\bar{\eta}}, \mathbb{Q}_{\ell}\right) \subseteq$ $\mathrm{N}^{n} H^{2 n+1}\left(X_{\overline{0}}, \mathbb{Q}_{\ell}\right)$. Thus we have a commutative diagram

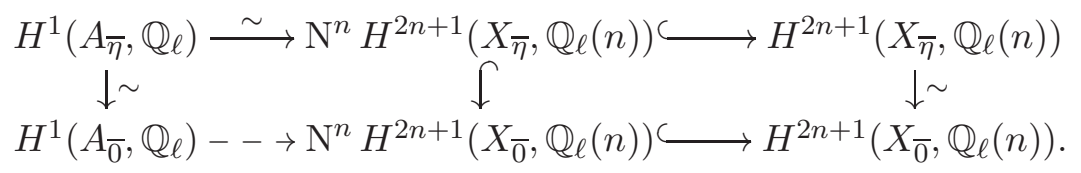

A dimension count implies that the induced dashed arrow is an isomorphism. Finally, since specialization is compatible with cup product in cohomology, the dashed arrow is in fact induced by the specialization of the correspondence inducing (6.1).

(c) follows from (b).

As an immediate consequence, we find:

Corollary 6.2. With $X / S$ as in Lemma 6.1, suppose further that $\operatorname{char}(K)=\operatorname{char}(\kappa)=0$ and that $\operatorname{dim} \mathrm{N}^{1} H^{3}\left(X_{\bar{\eta}}, \mathbb{Q}_{\ell}\right)=\operatorname{dim} \mathrm{N}^{1} H^{3}\left(X_{\overline{0}}, \mathbb{Q}_{\ell}\right)$. Then $\underline{\mathrm{Ab}}^{2}\left(X_{\bar{\eta}}\right)_{0}$ (Theorem 4.4 and Remark 4.5) and $\underline{\mathrm{Ab}}^{2}\left(X_{\overline{0}}\right)$ are isogenous abelian varieties over $\kappa$.

Proof. Use Lemma 6.1 and Corollary 5.2 .

Remark 6.3. If one assumes further in the corollary that $\phi_{X_{\bar{\eta}}}^{2}$ and $\phi_{X_{\overline{0}}}^{2}$ are isomorphisms (e.g., by [BS83. Th. 1(i)], if $\mathrm{CH}_{0}\left(X_{\bar{\eta}}\right)$ and $\mathrm{CH}_{0}\left(X_{\overline{0}}\right)$ are supported on curves after any base-change of algebraically closed field), then one may conclude in fact that $\underline{\mathrm{Ab}}^{2}\left(X_{\bar{\eta}}\right)_{0}$ and $\underline{\mathrm{Ab}}^{2}\left(X_{\overline{0}}\right)$ are isomorphic.

In the setting of Mazur's original question (over a number field $K$ ), the following corollary shows that under the geometric coniveau hypothesis there is an abelian variety over $K$ which interpolates the isogeny classes described by Honda-Tate theory.

Corollary 6.4. Let $S$ be a Dedekind scheme with generic point $\eta$. Let $X \rightarrow S$ be a smooth projective morphism, and let $A_{\eta} / \eta$ be a phantom abelian variety for $X_{\eta}$ in degree $2 n+1$ (i.e., (6.1) is an isomorphism). Then $A_{\eta}$ extends to an abelian scheme $A / S$ such that, for each point $s \in S, A_{s}$ is a phantom abelian variety for $X_{s}$ in degree $2 n+1$.

Proof. Let $A$ be the Néron model of $A$ over $S$. Lemma 6.1 shows that every fiber is an abelian variety (and thus $A$ is an abelian scheme), and that each fiber is a phantom for the corresponding fiber of $X$.

However, like the Néron-Severi group, the rank of the geometric coniveau $n$ piece in degree $2 n+1$ can vary in families; this behavior rules out the existence, in general, of a relative phantom abelian scheme modeling the coniveau $n$ piece in degree $2 n+1$ for families of projective varieties. In particular, algebraic representatives need not specialize to algebraic representatives. The following provides such an example:

Example 6.5. Let $S=\operatorname{Spec} R$ be the spectrum of a discrete valuation ring as in Lemma 6.1, and let $E$ be a CM field such that $[E: \mathbb{Q}]=6$ and $\left[\widetilde{E}: \widetilde{E^{(+)}}\right]=8$, where $\widetilde{E}$ and $\widetilde{E^{(+)}}$are the normal closures over $\mathbb{Q}$ of $E$ and its maximal totally real subfield, respectively. Let $X / S$ be an 
abelian threefold such that $\operatorname{End}\left(X_{\bar{\eta}}\right) \cong \mathbb{Z}$ and $\operatorname{End}\left(X_{0}\right) \cong \operatorname{End}\left(X_{\overline{0}}\right) \supseteq \mathcal{O}_{E}$. On one hand, Tan93, Thm. 1] implies that $\operatorname{dim} \mathrm{N}^{1} H^{3}\left(X_{\bar{\eta}}, \mathbb{Q}_{\ell}\right)=6$. On the other hand, Tan93, Thm. 2] implies that if $\operatorname{char}(\kappa)=0$, then $\operatorname{dim} \mathrm{N}^{1} H^{3}\left(X_{\overline{0}}, \mathbb{Q}_{\ell}\right)=18$, while if $\operatorname{char}(\kappa)>0$ a further specialization argument guarantees that $\operatorname{dim} \mathrm{N}^{1} H^{3}\left(X_{\overline{0}}, \mathbb{Q}_{\ell}\right) \geq 18$. Thus there is no abelian scheme $J / S$ so that for each $s \in S$, there is an isomorphism $H^{1}\left(J_{\bar{s}}, \mathbb{Q}_{\ell}\right) \cong \mathrm{N}^{1} H^{3}\left(X_{\bar{s}}, \mathbb{Q}_{\ell}\right)$. Moreover, if $\operatorname{char}(\kappa)=0$, this gives an example where $\underline{\mathrm{Ab}}^{2}\left(X_{\bar{\eta}}\right)_{0}$ is not isogenous to $\underline{\mathrm{Ab}}^{2}\left(X_{\overline{0}}\right)$.

6.2. Algebraic representatives for higher codimension cycles. In order to employ Theorem 5.1. one must first have an algebraic representative. However, outside of the cases of codimension-1, codimension-2, and codimension- $d(=\operatorname{dim} X)$ cycles, there are not many results on their existence. Saito's result [Sai79, Thm. 2.2] (see Proposition 3.2) provides a criterion for establishing the existence of algebraic representatives. In this section, we use this to provide some further criteria for when algebraic representatives exist. To begin, Proposition 6.6 suggested by the referee, asserts that if a variety is dominated by a second variety that admits an algebraic representative, then the first variety also admits an algebraic representative. Next, Proposition 6.7 (see also Remark 6.8) shows that the existence of algebraic representatives for codimension-3 cycles is a birational invariant.

Proposition 6.6. Let $K$ be a field, $n$ be a natural number, and let $f: Y \rightarrow X$ be a dominant morphism of smooth projective varieties over $K$. Assume there exists an algebraic representative for $\mathrm{A}^{n}\left(Y_{\bar{K}}\right)$. Then there exists an algebraic representative for $\mathrm{A}^{n}\left(X_{\bar{K}}\right)$.

Proof. Let $\iota: Z \hookrightarrow Y$ be a closed subvariety of pure dimension $\operatorname{dim} X$ cut out by hyperplanes. The morphism $\left.f\right|_{Z}: Z \rightarrow X$ is then generically finite, of degree $r$, say. Assume there exists an algebraic representative $\mathrm{Ab}^{n}\left(Y_{\bar{K}}\right)$ for $\mathrm{A}^{n}\left(Y_{\bar{K}}\right)$. By the projection formula, the induced map

$$
\left(\left.f\right|_{Z}\right)_{*}\left(\left.f\right|_{Z}\right)^{*}=\left(\left.f\right|_{Z}\right)_{*} \iota^{*} f^{*}: \mathrm{A}^{n}\left(X_{\bar{K}}\right) \rightarrow \mathrm{A}^{n}\left(X_{\bar{K}}\right)
$$

is multiplication by $r$. This map is surjective because $\mathrm{A}^{n}\left(X_{\bar{K}}\right)$ is a divisible group. It follows that the map

$$
\left(\left.f\right|_{Z}\right)_{*} \iota^{*}: \mathrm{A}^{n}\left(Y_{\bar{K}}\right) \rightarrow \mathrm{A}^{n}\left(X_{\bar{K}}\right)
$$

is surjective. Consider then a surjective regular homomorphism $\mathrm{A}^{n}\left(X_{\bar{K}}\right) \rightarrow A(\bar{K})$ to an abelian variety $A$ defined over $\bar{K}$. Then the composition

$$
\mathrm{A}^{n}\left(Y_{\bar{K}}\right) \stackrel{\left(\left.f\right|_{Z}\right)_{*} \iota^{*}}{\longrightarrow} \mathrm{A}^{n}\left(X_{\bar{K}}\right) \longrightarrow A(\bar{K})
$$

is a surjective regular homomorphism. By the universal property of the algebraic representative $\mathrm{Ab}^{n}\left(Y_{\bar{K}}\right)$ for $\mathrm{A}^{n}\left(Y_{\bar{K}}\right)$, we find that $\operatorname{dim} A \leq \operatorname{dim} \mathrm{Ab}^{n}\left(Y_{\bar{K}}\right)$. By [Sai79, Thm. 2.2] (see Proposition 3.2), it follows that $\mathrm{A}^{n}\left(X_{\bar{K}}\right)$ admits an algebraic representative.

Proposition 6.7. Let $K \subseteq \Omega$ be a sub-field of a universal domain $\Omega$ and let $X$ and $Y$ be smooth projective varieties over $K$ with a correspondence $\Gamma \in \mathrm{CH}^{d}(Y \times X)$ such that $\left(\Gamma_{\Omega}\right)_{*}: \mathrm{CH}_{0}\left(Y_{\Omega}\right)_{\mathbb{Q}} \rightarrow$ $\mathrm{CH}_{0}\left(X_{\Omega}\right)_{\mathbb{Q}}$ is surjective. Assume that $\mathrm{A}^{3}\left(Y_{\bar{K}}\right)$ admits an algebraic representative (e.g. if $\operatorname{dim} Y \leq$ 3). Then $\mathrm{A}^{3}\left(X_{\bar{K}}\right)$ admits an algebraic representative.

Proof. The assumption that $\mathrm{CH}_{0}\left(X_{\Omega}\right)$ is spanned by $\mathrm{CH}_{0}\left(Y_{\Omega}\right)$, via the action of the correspondence $\Gamma$, implies by a decomposition of the diagonal argument [BS83, Prop. 1] (see also [Via, Prop. 3.5] for the factorization assertion below) the existence of a positive integer $N$ such that $N \Delta_{X}=Z_{1}+Z_{2}$ in $\mathrm{CH}^{d}(X \times X)$, with $Z_{1}$ supported on $D \times X$ for some divisor $D \subset X$, and $Z_{2}$, when seen as a correspondence from $X$ to $X$, factoring as $\Gamma \circ \Gamma^{\prime}$ for some correspondence $\Gamma^{\prime} \in \mathrm{CH}_{d}(X \times Y)$. Let us denote $\widetilde{D}$ an alteration of $D$ and $\iota: \widetilde{D} \rightarrow D \rightarrow X$ the natural morphism. 
Consider a surjective regular homomorphism $\mathrm{A}^{3}\left(X_{\bar{K}}\right) \rightarrow A(\bar{K})$. We see from the divisibility of $\mathrm{A}^{3}\left(X_{\bar{K}}\right)$ and from the decomposition of the diagonal that

$$
\mathrm{A}^{3}\left(X_{\bar{K}}\right)=\left(N \Delta_{X}\right)^{*} \mathrm{~A}^{3}\left(X_{\bar{K}}\right)=\left(\Gamma^{\prime}\right)^{*} \mathrm{~A}^{3}\left(Y_{\bar{K}}\right)+\iota_{*} \mathrm{~A}^{2}\left(\widetilde{D}_{\bar{K}}\right) .
$$

It follows that the regular homomorphism

$$
\mathrm{A}^{3}\left(Y_{\bar{K}}\right) \oplus \mathrm{A}^{2}\left(\widetilde{D}_{\bar{K}}\right) \stackrel{\left(\Gamma^{\prime}\right)^{*} \oplus \iota_{*}}{\longrightarrow} \mathrm{A}^{3}\left(X_{\bar{K}}\right) \longrightarrow A(\bar{K})
$$

is surjective. Since both $\mathrm{A}^{3}\left(Y_{\bar{K}}\right)$ and $\mathrm{A}^{2}\left(\widetilde{D}_{\bar{K}}\right)$ admit an algebraic representative (by assumption and by Murre's Theorem 3.4, respectively), we get by the criterion of [Sai79, Thm. 2.2] (see Proposition 3.2) that there exists an integer $M$ independent of the surjective regular homomorphism $\mathrm{A}^{3}\left(X_{\bar{K}}\right) \rightarrow$ $A(\bar{K})$ such that $\operatorname{dim} A \leq M$. We conclude, by the same criterion, that $\mathrm{A}^{3}\left(X_{\bar{K}}\right)$ has an algebraic representative.

Remark 6.8. The existence of an algebraic representative for codimension-3 algebraically trivial cycles is a birational invariant of smooth projective varieties over $\bar{K}$. Indeed, let $f: Y \rightarrow X$ be a dominant map of smooth projective varieties over $\bar{K}$. Then $f_{*}: \mathrm{CH}_{0}\left(Y_{\Omega}\right) \rightarrow \mathrm{CH}_{0}\left(X_{\Omega}\right)$ is surjective, and it follows from Proposition 6.7 that, if $\mathrm{A}^{3}(Y)$ has an algebraic representative, then $\mathrm{A}^{3}(X)$ has an algebraic representative.

Remark 6.9. Proposition 6.7 can be generalized by repeated use of the decomposition of the diagonal argument. Specifically, one can show that if $X$ is a smooth projective variety over $K$ such that $\mathrm{CH}_{0}\left(X_{\Omega}\right)_{\mathbb{Q}}, \ldots, \mathrm{CH}_{l}\left(X_{\Omega}\right)_{\mathbb{Q}}$ are spanned by the $\mathrm{CH}_{0}$ of threefolds via the action of correspondences, then $\mathrm{A}^{0}\left(X_{\bar{K}}\right), \ldots, \mathrm{A}^{l+3}\left(X_{\bar{K}}\right)$ admit algebraic representatives.

\section{REFERENCES}

[ACMVa] J. Achter, S. Casalaina-Martin, and Ch. Vial, Algebraic representatives over arbitrary fields, in preparation.

[ACMVb] _ On descending cohomology geometrically : quadric fibrations, in preparation.

[ACMVc] - Parameter spaces for algebraic equivalence, preprint, arXiv:1610.06586, arXiv:1610.06586.

[And04] Yves André, Une introduction aux motifs (motifs purs, motifs mixtes, périodes), Panoramas et Synthèses [Panoramas and Syntheses], vol. 17, Société Mathématique de France, Paris, 2004. MR 2115000 (2005k:14041)

[Bea77] Arnaud Beauville, Variétés de Prym et jacobiennes intermédiaires, Ann. Sci. École Norm. Sup. (4) 10 (1977), no. 3, 309-391. MR 0472843 (57 \#12532)

[Blo79] S. Bloch, Torsion algebraic cycles and a theorem of Roitman, Compositio Math. 39 (1979), no. 1, $107-127$. MR 539002 (80k:14012)

[Blo10] L L Lectures on algebraic cycles, second ed., New Mathematical Monographs, vol. 16, Cambridge University Press, Cambridge, 2010. MR 2723320 (2011h:14009)

[BLR90] Siegfried Bosch, Werner Lütkebohmert, and Michel Raynaud, Néron models, Ergebnisse der Mathematik und ihrer Grenzgebiete (3) [Results in Mathematics and Related Areas (3)], vol. 21, Springer-Verlag, Berlin, 1990. MR 1045822 (91i:14034)

[BS83] S. Bloch and V. Srinivas, Remarks on correspondences and algebraic cycles, Amer. J. Math. 105 (1983), no. 5, 1235-1253. MR 714776 (85i:14002)

[Con06] Brian Conrad, Chow's $K / k$-image and $K / k$-trace, and the Lang-Néron theorem, Enseign. Math. (2) 52 (2006), no. 1-2, 37-108. MR 2255529 (2007e:14068)

[Del72] Pierre Deligne, Les intersections complètes de niveau de Hodge un, Invent. Math. 15 (1972), $237-250$. MR MR0301029 (46 \#189)

[Del80] _ La conjecture de Weil. II, Inst. Hautes Études Sci. Publ. Math. (1980), no. 52, $137-252$. MR 601520 (83c:14017)

[Del82] Hodge cycles on abelian varieties (notes by J. Milne), Hodge cycles, motives, and Shimura varieties (Pierre Deligne, James S. Milne, Arthur Ogus, and Kuang-yen Shih, eds.), Lecture Notes in Mathematics, vol. 900, Springer-Verlag, Berlin-New York, 1982, pp. ii+414. MR 654325 (84m:14046)

[Fal83] G. Faltings, Endlichkeitssätze für abelsche Varietäten über Zahlkörpern, Invent. Math. 73 (1983), no. 3, 349-366. MR 718935 (85g:11026a) 
[Fri91] Eric M. Friedlander, Algebraic cycles, Chow varieties, and Lawson homology, Compositio Math. 77 (1991), no. 1, 55-93. MR 1091892 (92a:14005)

[Ful98] William Fulton, Intersection theory, second ed., Ergebnisse der Mathematik und ihrer Grenzgebiete. 3. Folge. A Series of Modern Surveys in Mathematics [Results in Mathematics and Related Areas. 3rd Series. A Series of Modern Surveys in Mathematics], vol. 2, Springer-Verlag, Berlin, 1998. MR 1644323 (99d:14003)

[FWG ${ }^{+}$92] Gerd Faltings, Gisbert Wüstholz, Fritz Grunewald, Norbert Schappacher, and Ulrich Stuhler, Rational points, third ed., Aspects of Mathematics, E6, Friedr. Vieweg \& Sohn, Braunschweig, 1992, Papers from the seminar held at the Max-Planck-Institut für Mathematik, Bonn/Wuppertal, 1983/1984, With an appendix by Wüstholz. MR 1175627 (93k:11060)

[Gab83] Ofer Gabber, Sur la torsion dans la cohomologie l-adique d'une variété, C. R. Acad. Sci. Paris Sér. I Math. 297 (1983), no. 3, 179-182. MR 725400 (85f:14018)

[Gro68] Alexander Grothendieck, Le groupe de Brauer. III. Exemples et compléments, Dix Exposés sur la Cohomologie des Schémas, North-Holland, Amsterdam; Masson, Paris, 1968, pp. 88-188. MR 0244271 (39 \#5586c)

[GW10] Ulrich Görtz and Torsten Wedhorn, Algebraic geometry I, Advanced Lectures in Mathematics, Vieweg + Teubner, Wiesbaden, 2010, Schemes with examples and exercises. MR 2675155 (2011f:14001)

[Hon68] Taira Honda, Isogeny classes of abelian varieties over finite fields, J. Math. Soc. Japan 20 (1968), 83-95. MR 0229642 (37 \#5216)

[Ill06] Luc Illusie, Miscellany on traces in $\ell$-adic cohomology: a survey, Jpn. J. Math. 1 (2006), no. 1, $107-136$. MR 2261063 (2007g:14016)

[Jan94] Uwe Jannsen, Motivic sheaves and filtrations on Chow groups, Motives (Seattle, WA, 1991), Proc. Sympos. Pure Math., vol. 55, Amer. Math. Soc., Providence, RI, 1994, pp. 245-302. MR 1265533 (95c:14006)

[Kle68] S. L. Kleiman, Algebraic cycles and the Weil conjectures, Dix exposés sur la cohomologie des schémas, North-Holland, Amsterdam; Masson, Paris, 1968, pp. 359-386. MR 0292838 (45 \#1920)

[Kol96] János Kollár, Rational curves on algebraic varieties, Ergebnisse der Mathematik und ihrer Grenzgebiete. 3. Folge. A Series of Modern Surveys in Mathematics [Results in Mathematics and Related Areas. 3rd Series. A Series of Modern Surveys in Mathematics], vol. 32, Springer-Verlag, Berlin, 1996. MR 1440180 (98c:14001)

[Lec86] Florence Lecomte, Rigidité des groupes de Chow, Duke Math. J. 53 (1986), no. 2, 405-426. MR 850543 (88c:14013)

[Maz72] B. Mazur, Frobenius and the Hodge filtration, Bull. Amer. Math. Soc. 78 (1972), 653-667. MR 0330169 (48 \#8507)

[Maz11] _ For the "open problem session" in the conference in honor of Joe Harris, unpublished note, 2011.

[Maz14] O Open problems: Descending cohomology, geometrically, Notices of the International Congress of Chinese Mathematicians 2 (2014), no. 1, $37-40$.

[Mil94] J. S. Milne, Motives over finite fields, Motives (Seattle, WA, 1991), Proc. Sympos. Pure Math., vol. 55, Amer. Math. Soc., Providence, RI, 1994, pp. 401-459. MR 1265538 (95g:11053)

[Mil14] _ Algebraic geometry (v6.00), 2014, Available at www.jmilne.org/math/, p. 223.

[Moc12] Shinichi Mochizuki, Topics in absolute anabelian geometry I: generalities, J. Math. Sci. Univ. Tokyo 19 (2012), no. 2, 139-242. MR 2987306

[Mur85] J. P. Murre, Applications of algebraic K-theory to the theory of algebraic cycles, Algebraic geometry, Sitges (Barcelona), 1983, Lecture Notes in Math., vol. 1124, Springer, Berlin, 1985, pp. 216-261. MR 805336 (87a:14006)

[Poo04] Bjorn Poonen, Bertini theorems over finite fields, Ann. of Math. (2) 160 (2004), no. 3, $1099-1127$. MR 2144974 (2006a:14035)

[Sai79] Hiroshi Saito, Abelian varieties attached to cycles of intermediate dimension, Nagoya Math. J. 75 (1979), 95-119. MR 542191 (81a:14021)

[Sam60] Pierre Samuel, Relations d'équivalence en géométrie algébrique, Proc. Internat. Congress Math. 1958, Cambridge Univ. Press, New York, 1960, pp. 470-487. MR 0116010

[Tan93] S. G. Tankeev, Abelian varieties and the general Hodge conjecture, Izv. Ross. Akad. Nauk Ser. Mat. 57 (1993), no. 4, 192-206. MR 1243359 (95j:14011)

[Tat66] John Tate, Endomorphisms of abelian varieties over finite fields, Invent. Math. 2 (1966), $134-144$. MR 0206004 (34 \#5829)

[Tat71] Classes d'isogénie des variétés abéliennes sur un corps fini (d'après T. Honda), Séminaire Bourbaki. Vol. 1968/69: Exposés 347-363, Lecture Notes in Math., vol. 175, Springer, Berlin, 1971, pp. Exp. No. 352, 95-110. MR 3077121

[Via] Charles Vial, Remarks on motives of abelian type, Tohoku Math. J., in press. 
[Via13] Niveau and coniveau filtrations on cohomology groups and Chow groups, Proc. Lond. Math. Soc. (3) 106 (2013), no. 2, 410-444. MR 3021467

[Voi04] Claire Voisin, Remarks on filtrations on Chow groups and the Bloch conjecture, Ann. Mat. Pura Appl. (4) 183 (2004), no. 3, 421-438. MR 2082666

[Voi07] Hodge theory and complex algebraic geometry. I, english ed., Cambridge Studies in Advanced Mathematics, vol. 76, Cambridge University Press, Cambridge, 2007, Translated from the French by Leila Schneps. MR 2451566 (2009j:32014)

[Voi13] Abel-Jacobi map, integral Hodge classes and decomposition of the diagonal, J. Algebraic Geom. 22 (2013), no. 1, 141-174. MR 2993050

[Voi14] Chow rings, decomposition of the diagonal, and the topology of families, Annals of Mathematics Studies, vol. 187, Princeton University Press, Princeton, NJ, 2014. MR 3186044

[Voi15] Unirational threefolds with no universal codimension 2 cycle, Invent. Math. 201 (2015), no. 1, 207-237. MR 3359052

[Vol05] Maja Volkov, A class of p-adic Galois representations arising from abelian varieties over $\mathbb{Q}_{p}$, Math. Ann. 331 (2005), no. 4, 889-923. MR 2148801 (2006a:14027)

[Wei54] André Weil, Sur les critères d'équivalence en géométrie algébrique, Math. Ann. 128 (1954), 95-127. MR 0065219 (16,398e)

[Zar75] Ju. G. Zarhin, Endomorphisms of Abelian varieties over fields of finite characteristic, Izv. Akad. Nauk SSSR Ser. Mat. 39 (1975), no. 2, 272-277, 471. MR 0371897 (51 \#8114)

[Zar08] Yuri G. Zarhin, Homomorphisms of abelian varieties over finite fields, Higher-dimensional geometry over finite fields, NATO Sci. Peace Secur. Ser. D Inf. Commun. Secur., vol. 16, IOS, Amsterdam, 2008, pp. 315343. MR 2484084 (2010a:11117)

Colorado State University, Department of Mathematics, Fort Collins, CO 80523, USA

E-mail address: j.achter@colostate.edu

University of Colorado, Department of Mathematics, Boulder, CO 80309, USA

E-mail address: casa@math.colorado.edu

University of CAmbridge, DPMMS, CAmbridge CB3 0WB, UK

E-mail address: c.vial@dpmms.cam.ac.uk 